\title{
Optimum adjuvant trastuzumab duration for human epidermal growth factor receptor-2 positive breast cancer: a network meta-analysis of randomized trials
}

\author{
$\mathrm{Ji} \mathrm{Ma}^{1 \#}$, Xiaofang Tang ${ }^{2 \#}$, Qiancheng $\mathrm{Hu}^{1}$, Qingfeng Wang ${ }^{1}$, Ye Chen ${ }^{1}$, Xiaofen $\mathrm{Li}^{1}$, Ting $\mathrm{Luo}^{3,4}$, Dan Cao ${ }^{1}$ \\ ${ }^{1}$ Department of Abdominal Oncology, Cancer Center, West China Hospital, Sichuan University, Chengdu, China; ${ }^{2}$ Department of Emergency, \\ Disaster Medical Center, West China Hospital, Sichuan University, Chengdu, China; ${ }^{3}$ Department of Head, Neck and Mammary Gland Oncology, \\ Cancer Center, West China Hospital, Sichuan University, Chengdu, China; ${ }^{4}$ Laboratory of Molecular Diagnosis of Cancer, Clinical Research Center \\ for Breast, West China Hospital, Sichuan University, Chengdu, China \\ Contributions: (I) Conception and design: T Luo, D Cao; (II) Administrative support: D Cao; (III) Provision of study materials or patients: Q Hu, J \\ Ma; (IV) Collection and assembly of data: Q Hu, J Ma; (V) Data analysis and interpretation: X Tang, Q Hu; (VI) Manuscript writing: All authors; (VII) \\ Final approval of manuscript: All authors. \\ "These authors contributed equally to this work. \\ Correspondence to: Ting Luo, MD. Department of Head, Neck and Mammary Gland Oncology, Cancer Center, Sichuan University, No. 37 Guo \\ Xue Xiang, Chengdu 610041, China. Email: tina621@163.com; Dan Cao, MD. Department of Abdominal Oncology, Cancer Center West China \\ Hospital, Sichuan University, No. 37 Guo Xue Xiang, Chengdu 610041, China. Email: caodan316@163.com.
}

\begin{abstract}
Background: Adjuvant trastuzumab treatment for 12 months is the standard-of-care for early HER2positive breast cancer; however, the optimal duration is unclear. We performed a network meta-analysis (NMA) to determine the optimal treatment duration.

Methods: We identified 16 randomized controlled trials involving 29,837 patients that assessed trastuzumab treatment in HER2-positive early breast cancer. Our NMA compared six trastuzumab durations: observation, T-9 weeks, T-12 weeks, T-6 months, T-12 months, and T-24 months. We assessed overall survival (OS), disease-free survival (DFS), acceptability, and cardiotoxicities and grade 3-4 nonhematologic toxicities, and ranked the durations in terms of efficacy and safety by surface under the cumulative ranking (SUCRA).

Results: Pairwise meta-analysis showed that while T-6 months was associated with a significant reduction in DFS compared to T-12 months. In our NMA, increasing or decreasing durations showed a significant benefit in DFS compared to observation; however, decreasing durations was not associated with a significant reduction in DFS compared with T-12 months, regardless of the lymph node and hormone receptor statuses. SUCRA ordered the optimum durations of trastuzumab treatment based on PFS as T-12 months (95.6\%), T-24 months (69.6\%), T-6 months (53.2\%), T-9 weeks (41.2\%), T-12 weeks (34.3\%) and observation (6.1\%).

Conclusions: Escalating trastuzumab treatment beyond T-12 months confers no additional survival benefit but increased risk of cardiotoxicities. Furthermore, de-escalating treatment confers no improvement on OS compared to T-12 months. These data suggest that T-12 months is the most appropriate treatment schedule for HER2-positive early breast cancer.
\end{abstract}

Keywords: Early breast cancer; trastuzumab; network meta-analysis (NMA)

Submitted Jun 21, 2020. Accepted for publication Mar 05, 2021.

doi: $10.21037 /$ tcr-20-2378

View this article at: http://dx.doi.org/10.21037/tcr-20-2378

\section{Introduction}

Human epidermal growth factor receptor-2 (HER2)- positive breast cancer accounts for $\sim 20-25 \%$ of all breast cancers $(1,2)$ and the overall prognosis of HER2-positive breast cancer is poor compared to other breast cancer 
subtype (3). Trastuzumab is a monoclonal antibody which specifically targets the extracellular domain of HER2 (4). Several pivotal phase III clinical trials have proved that a 12 -month (T-12) treatment duration is superior to observation (4-7), leading to national and international guidelines to recommend $\mathrm{T}-12$ months treatment with or after chemotherapy as the standard-of-care (8-10). A shorter duration of trastuzumab is associated with reduced side effects and costs (11), the optimum duration of trastuzumab treatment may be hampered by efficacy, toxicity, convenience, and cost (12).

High-quality randomized controlled trials (RCTs) have aimed to evaluate the optimum duration of trastuzumab treatment (3-6,12). For example, the HERA trial showed that trastuzumab treatment for 24 months (T-24 months) did not improve disease-free survival (DFS) compared to treatment for T-12 months [hazard ratio (HR) 1.02, 95\% confidence interval (CI): 0.89-1.17], and was associated with higher costs, inconvenience, and cardiac toxicity (7.3\% vs. 4.4\%) (12). The PERSEPHONE trial compared T-12 months trastuzumab treatment with 6 months (T-6 months) treatment, and found that T-12 months was non-inferior and decreased cardiac toxicity $(8 \% v s$. $4 \%, \mathrm{P}<0.001)(13)$. Conversely, the PHARE and HORG trials failed to show that T-12 months was non-inferior compared to T-6 months (14-16). Finally, the SOLD and Short-HER trials found that trastuzumab treatment for just 9 weeks (T-9 weeks) was inferior to T-12 months, but conferred fewer cardiac adverse effects $(17,18)$. Together with these conflicting results, a considerable gap exists in the current literature as a large proportion of the RCTs have compared active therapy to inactive interventions (e.g., observation) $(5,6,12)$ rather than comparing different treatment durations. De-escalating trastuzumab treatment in HER2-positive early breast cancer has attracted the attention of many investigators (9). Previous studies also showed that T12-month trastuzumab treatment prevents disease recurrence and confers survival benefits for patients with HER2-positive early breast cancer compared with observation (19). Five important systematic reviews attempted to resolve the debate over de-escalating treatment and standard care, and the results undoubtedly concluded that T-6 months could not replace T-12 months in patients with HER2-positive early breast cancer based on inferior survival $(12,14-17)$. However, these studies failed to make sufficient comparisons between de-escalating treatments and standard care in terms of survival and toxicities to draw firm conclusions. Finally, there is no information on de-escalating and escalating trastuzumab treatment in patients with HER2-positive early breast cancer; thus, there is heated controversy about whether escalating and deescalating treatment might be considered a new standard of care.

This study used a network meta-analysis (NMA) approach (18) to evaluate multiple interventions in a single analysis and the clinical outcomes as a result of escalating, de-escalating and standard (T-12 months) trastuzumab treatments. Specifically, we synthesized all available evidence from 16 RCTs based on the direct and indirect comparisons of trastuzumab efficacy and safety to identify the optimum treatment duration (T-24 months vs. T-12 months vs. T-6 months vs. T-12 weeks vs. T-9 weeks vs. observation) with the greatest clinical value in HER2-positive, early breast cancers. This study was performed in line with the Preferred Reporting Items for Systematic Reviews and Meta-Analyses (PRISMA) reporting checklist $(20,21)$ (available at http://dx.doi.org/10.21037/tcr-20-2378).

\section{Methods}

A detailed protocol has been registered in PROSPERO (CRD42019139109) and published in BMJ Open recently (22).

\section{Search strategy}

Titles and abstracts referring to trastuzumab treatment for early breast cancers were searched in the electronic PubMed, Cochrane Library and Embase (Ovid interface) databases from conception to June 16, 2019. Titles and abstracts were also searched in international meeting proceedings, including the San Antonio Breast Cancer Symposium (SABCS) (2015 to 2019), European Society of Medical Oncology (ESMO) and the American Society of Clinical Oncology (ASCO).

Two reviewers trained in data extraction conducted the searches, independently. Pairs of reviewers then manually searched the reference lists from eligible reviews and relevant RCTs to identify additional potential studies for inclusion. The reasons for excluding a full-text article were recorded and a PRISMA flow diagram for the NMA was generated.

The search terms included the following domains of the Medical Subject Heading $(\mathrm{MeSH})$ terms: 'breast cancer', 'human epidermal growth factor receptor-2' and 'trastuzumab', according to the Population Intervention Comparison Outcomes Study Design (PICOS) statement. 
These MeSHs and subheadings were combined with 'AND' or 'OR'.

A pilot test was performed to evaluate inter-rater reliability and to adjust each screening stage: title and abstract, followed by full-text screening. Then, independent reviewers screened the titles and abstracts of related RCTs studies based on the inclusion and exclusion criteria (see below). The eligible or potentially eligible trials were assessed by reading the full texts when necessary. Any disagreements over data extraction were resolved by discussion or the other reviewer.

\section{Data extraction and management}

Literature search records were maintained in EndNote X7 (Thomson Reuters, CA, USA). Microsoft Excel 2010 (Microsoft Corp, Redmond, WA, www.microsoft.com) was used to collect outcomes of interest, including the first author, study design, recruitment time frame, interventions, sample size, and study endpoints (OS, DFS, acceptability, and cardiac and grade 3-4 nonhematologic toxicities).

\section{Inclusion criteria}

Trials were eligible if they met the following criteria: (I) population: HER2-positive early breast cancer patients of any age or ethnicity, treated with trastuzumab; (II) Interventions and Comparators: any duration of trastuzumab treatment; (III) outcomes: OS, DFS, acceptability, and cardiotoxicities and grade 3-4 nonhematologic toxicities; (IV) study design: RCTs; (V) language and other limitations: studies published in English language without date limitations. There were no restrictions on patient performance status (PS), or nationality.

RCTs involving interventions using trastuzumab biosimilars or combined palliative care with trastuzumab were excluded. Reviews, posters, abstracts, editorials and case reports, retrospective and prospective cohort studies were also excluded.

\section{Outcomes}

The study outcomes were OS, DFS, acceptability, and cardiac and grade 3-4 nonhematologic toxicities. The Common Terminology Criteria for Adverse Events of the National Cancer Institute was used for cardiac toxicity grading. Cardiac toxicity was defined as an asymptomatic decline in the left ventricular ejection fraction (LVEF) to $\leq 45 \%$, an absolute drop of $10-15 \%$ in follow-up echocardiography, symptomatic congestive heart failure [New York Heart Association (NYHA) class III/IV] or cardiac death $(23,24)$. The relative effectiveness for each network comparison was calculated among all interventions as previous described $(25,26)$.

\section{Risk of bias}

The risk of bias of RCTs in the NMA was assessed using the following domains outlined by the Cochrane Collaboration (27): random sequence generation, allocation concealment, participant and personnel blinding, incomplete outcome data, selective outcome reporting, and other bias. Two authors independently reviewed the RCTs and reported a high risk of bias as "-", a low risk of bias as "+", or an unclear risk of bias "?". Any disagreements on the risk of bias were resolved by discussion or the third reviewer, if needed.

\section{Quality of evidence}

The quality of evidence produced by the NMA was assessed using a modified version of the Grades of Recommendation, Assessment, Development and Evaluation (GRADE) (28). The GRADE process was completed using GRADEprofiler software (version 3.6.1) with the following primary domains: risk of bias, imprecision, inconsistency, indirectness and publication bias (29). The GRADE evidence was then categorized into the following stages: (I) high, (II) moderate, (III) low and (IV) very low quality. The starting confidence level for each network estimate was high but decreased according to the evaluation of the primary domains. Any disagreements were resolved by discussion between the two reviewers, with the help of a third reviewer.

\section{Statistical analysis}

Traditional pairwise and NMA were performed, and all graphics for pairwise analyses and NMA were generated with Stata 13.0 (StataCorp, College Station, TX, USA) (30). To cross-compare all eligible interventions, NMAs for the outcomes of interest were conducted according to a Bayesian Markov chain Monte Carlo approach, as previously described (31), in WinBUGS version 1.4.3 (MRC Biostatistics Unit, Cambridge, UK). Results regarding OS and DFS were calculated as HRs with 95\% CIs. KaplanMeier curves were extracted using Getdata Graph Digitizer 
2.26 (www.getdata-graph-digitizer.com) and were calculated via summary statistics where necessary, as previously described (32). The WinBUGS code was described by Woods and his colleagues (33). Each posterior distribution of the model parameter was calculated by generating 10,000 iterations with a 5,000 burn-in and a thinning interval of 1 for each chain.

A loop-specific method was used to statistically evaluate inconsistencies between the direct and indirect comparisons (34). Both fixed-effects and random-effects models were run. Inconsistencies were assessed by comparing the deviation information criteria (DIC) statistics in the fitted consistency and inconsistency models (35). The convergence of the model was assessed by the potential scale reduction factor (PSRF), where PSRF closer to one indicated the better convergence (36). The results of the probability statements of the intervention effects were ranked using surface under the cumulative ranking (SUCRA).

The most effective interventions in terms of efficacy and safety were then further evaluated to interpret the relative effect of all comparisons. Risk-benefit analyses for efficacy and toxicity in each pairwise comparison was completed. A two-sided $\mathrm{P}<0.05$ was considered statistically significant. The degree of heterogeneity was estimated based on the magnitude of $\mathrm{I}^{2}$, which derived from the NMA models: $\mathrm{I}^{2}>50 \%$ supported high heterogeneity (37). In cases of high heterogeneity, a random-effects model was used; otherwise, a fixed-effects model was used. Publication bias was explored using a funnel plot.

\section{Subgroup and sensitivity analyses}

To explore whether particular breast cancer subtypes might be more or less appropriate for specific trastuzumab durations, breast cancers were stratified into the following groups: hormone receptor-positive, hormone receptornegative, lymph node-positive and lymph node-negative. Subgroup analyses were conducted by pairwise comparisons and NMA, regardless of the heterogeneity estimates. Sensitivity analyses were conducted based on hormone receptor and lymph node statuses.

\section{Results}

\section{Systematic literature review}

We identified 1,500 records from our database searches; 385 were duplicates, and 1,044 were excluded for not meeting the study criteria (see materials and methods) after screening the titles and abstracts. Of the remaining potential 71 full-text articles, 41 were excluded mostly because of the involvement of palliative care in the intervention $(n=12)$, trastuzumab-induced cardiotoxicity $(\mathrm{n}=7)$, a pairwise metaanalysis was performed $(\mathrm{n}=5)$, the study had a retrospective nature $(n=4)$ or for other reasons $(n=13)$. Ultimately, 30 articles corresponding to $16 \mathrm{RCTs}$ met the criteria for inclusion in our review $(4-7,13,19,38-54)$ (Figure 1).

\section{RCT characteristics}

The 16 eligible RCTs included a total of 29,837 patients (Table 1). These RCTs were published between 2005 and 2019, and the number of patients ranged from 227 to 4,118 per trial. All RCTs were included in the survival analysis and were suitable to analyze congestive heart failure rates in the recruited patients. We used updated survival data to collect HRs for DFS and OS with 95\% CIs in our NMA: HRs with $95 \%$ CIs were directly reported in 14 studies and could be estimated in two studies, but the remaining study did not provide an HR estimate for OS (NeoSphere).

\section{Network plot}

We performed a network plot of six comparisons for the outcomes of interest (Figure 2): observation, T-9 weeks, T-12 weeks, T-6 months, T-12 months, and T-24 months. Patients were randomized to receive one of the previously mentioned treatment options. Two loop-specific with no inconsistencies were found in the NMA (Figure S1). Two RCTs had three arm-based studies and the remaining was two arm-based studies. The size of the node and the thickness of solid lines were directly proportional to the number of patients and the number of interventions, respectively. Figure S2 showed that there were six mixed comparisons and eight indirect comparisons in our NMA (Figure S2).

\section{Risk of bias and quality assessment}

All RCTs included in the NMA showed a low risk of bias in terms of the allocation concealment, random sequence generation and incomplete outcome data. Although these studies were open-label clinical trials, the results of interest were measured objectively in each trial and had a low risk of bias for blinding participants and personnel. The bias risk for all other sources was low in our NMA (Figure S3). 


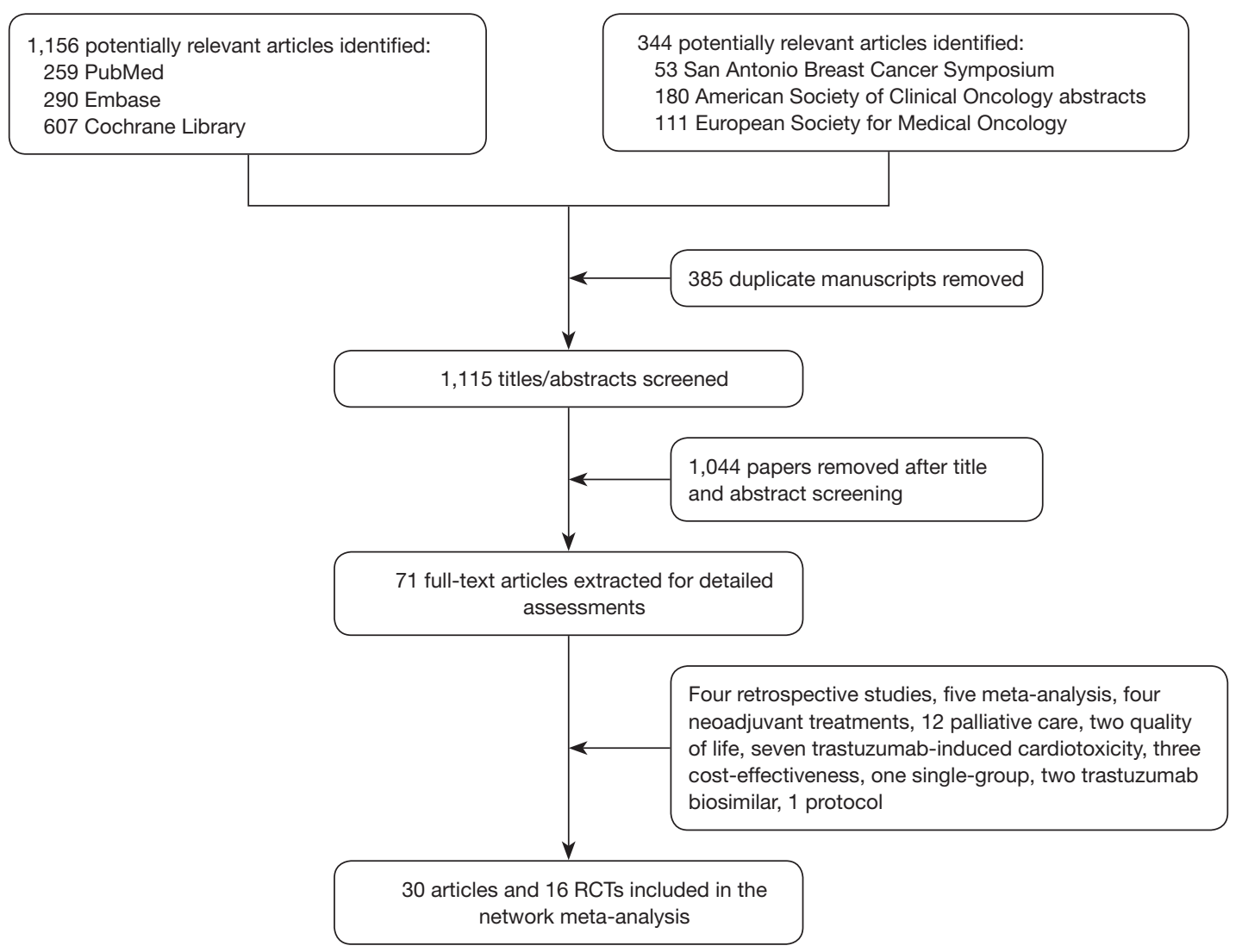

Figure 1 Literature search and selection.

In addition, funnel plot analysis did not detect any notable publication bias (Figure S4).

Finally, we assessed the quality of the outcomes of interest according to the GRADE criteria (Table S1). For most of the interventions, the quality of the primary endpoints of interest was determined to be either moderate or high.

\section{Pairwise meta-analysis}

All 16 RCTs included in our analysis reported information on DFS; only one trial did not report on OS. Here, we found that T-12 months was associated with a significant improvement in DFS compared to observation (HR 0.65, 95\% CI: $\left.0.59-0.70, \mathrm{I}^{2}=20.5 \%\right)$. Conversely, T-6 months was associated with a significant reduction in DFS compared with T-12 months (HR 1.15, 95\% CI: 1.01-1.28, $\left.\mathrm{I}^{2}=21.0 \%\right)$. Although not significant, the DFS conferred by T-12 months treatment was longer than the DFS conferred by T-9 weeks treatment (HR 1.25, 95\% CI: 1.00-1.50,
$\mathrm{I}^{2}=38.3 \%$ ) (Figure $3 A$ ). When comparing to observation, the OS data were consistent with the DFS data for T-12 months (HR 0.73, 95\% CI: 0.66-0.79, $\mathrm{I}^{2}=5.0 \%$ ). Neither T-6 months (HR 1.14, 95\% CI: 0.98-1.29, $\mathrm{I}^{2}=0.0 \%$ ) nor T-9 weeks (HR 1.20, 95\% CI: 0.90-1.50, $\left.\mathrm{I}^{2}=0.0 \%\right)$ significantly differed from $\mathrm{T}-12$ months in terms of OS (Figure 3B). Importantly, the differences between the traditional pairwise meta-analysis and the NMA were small, and there was consistency between direct and indirect evidence based on the comparisons of the results.

\section{$N M A$}

In our overall NMA, T-12 months, T-24 months, T-6 months, and T-9 weeks were all associated with significantly improved DFS compared to observation. Interestingly, no significant benefit was found between T-12 weeks and observation (HR 0.94, 95\% CI: 0.76-1.17). When comparing to T-12 months, both T-6 months and T-9 weeks were associated with significantly improved DFS 
Table 1 Characteristics of the studies included in this NMA

\begin{tabular}{|c|c|c|c|c|c|c|}
\hline Authors & Study identifier & $\mathrm{N}$ & $\begin{array}{l}\text { Recruitment } \\
\text { period }\end{array}$ & Primary endpoint & DFS HR (95\% Cl) & OS HR (95\% Cl) \\
\hline \multicolumn{7}{|c|}{$\begin{array}{l}\text { Studies comparing } \\
12 \text { months to observation }\end{array}$} \\
\hline \multirow[t]{2}{*}{ Slamon 2011} & BCIRG 006 & 3,222 & 2001-2004 & DFS & $0.68(0.56-0.83)^{\star}$ & $0.62(0.48-0.79)^{\star}$ \\
\hline & & & & & $0.81(0.66-0.98)^{\#}$ & $0.81(0.63-1.03)^{\#}$ \\
\hline Romond 2005 & NSABP B-31 & 2,119 & 2000-2005 & DFS & $0.59(0.50-0.68)$ & $0.66(0.54-0.79)$ \\
\hline Martine 2005 & HERA & 1,694 & 2001-2005 & DFS & $0.54(0.43-0.67)$ & $0.76(0.65-0.88)$ \\
\hline Marc 2009 & PACS 04 & 528 & 2001-2004 & Efficacy \& tolerance & $0.86(0.61-1.22)$ & $1.27(0.68-2.38)$ \\
\hline Gianni 2014 & $\mathrm{NOAH}$ & 235 & 2002-2005 & EFS & $0.64(0.44-0.93)$ & $0.66(0.43-1.01)$ \\
\hline Martine 2016 & ALTTO & 4,118 & $2007-2011$ & DFS & $0.63(0.52-0.75)$ & $0.76(0.56-1.02)$ \\
\hline \multicolumn{7}{|c|}{$\begin{array}{l}\text { Studies comparing } \\
24 \text { months to observation }\end{array}$} \\
\hline Martine 2005 & HERA & 1,693 & 2001-2005 & DFS & $0.77(0.69-0.87)$ & $0.74(0.63-0.86)$ \\
\hline \multicolumn{7}{|c|}{$\begin{array}{l}\text { Studies comparing } \\
9 \text { weeks to observation }\end{array}$} \\
\hline Joensuu 2006 & FinHer & 232 & 2000-2003 & RFS & $0.42(0.21-0.83)$ & $0.41(0.16-1.08)$ \\
\hline Earl 2019 & PERSEPHONE & 4,089 & $2007-2015$ & DFS & $1.17(0.93-1.24)$ & $1.14(0.95-1.37)$ \\
\hline Pivot 2019 & PHARE & 3,384 & 2006-2010 & DFS & $1.08(0.93-1.25)$ & $1.13(0.92-1.39)$ \\
\hline Mavroudis 2015 & HORG & 481 & 2004-2012 & DFS & $1.57(0.86-2.10)$ & $1.45(0.57-3.67)$ \\
\hline \multicolumn{7}{|c|}{$\begin{array}{l}\text { Studies comparing } \\
12 \text { weeks to } 12 \text { months }\end{array}$} \\
\hline Schneider 2015 & E2198 & 227 & 1999-2000 & Safety & $1.31(0.79-2.12)$ & $1.37(0.74-2.54)$ \\
\hline \multicolumn{7}{|c|}{$\begin{array}{l}\text { Studies comparing } \\
9 \text { weeks to } 12 \text { months }\end{array}$} \\
\hline Joensuu 2018 & SOLD & 2,176 & 2008-2014 & DFS & $1.39(1.12-1.72)$ & $1.36(0.98-1.89)$ \\
\hline Conte 2017 & ShortHER & 1,254 & 2007-2013 & DFS \& OS & 1.13 (0.89-1.42) & $1.07(0.74-1.56)$ \\
\hline
\end{tabular}

*, doxorubicin and cyclophosphamide, followed by docetaxel plus trastuzumab (AC-TH); ${ }^{*}$, docetaxel and carboplatin, given concurrently with trastuzumab, followed by trastuzumab $(\mathrm{TCH}) ;{ }^{\S}$, HRs were estimated from summary statistics with the method described by Tierney et al. N, number of patients; DFS, disease-free survival; OS, overall survival; HR, hazard ratio; PCR, pathological complete response; EFS, event-free survival; RFS, relapse-free survival; and NA, not applicable. 


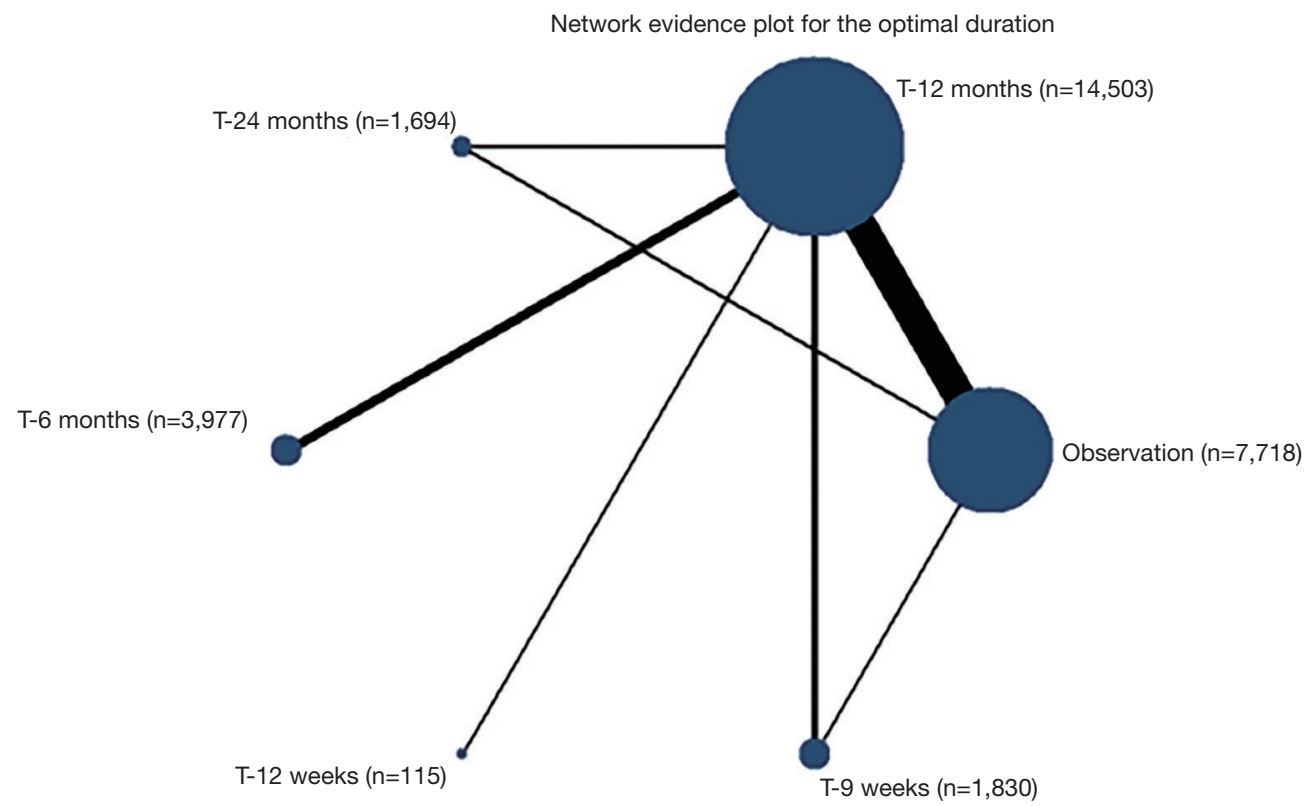

Figure 2 Network plot of the six comparisons for the outcomes of interest. The size of the node and the thickness of solid lines are directly proportional to the number of patients and the number of interventions, respectively. T, trastuzumab.

(Figure 4A). We produced similar findings for OS, with the exception that only T-12 months (HR 0.87, 95\% CI: 0.85-0.91) and T-24 months (HR 0.88, 95\% CI: 0.84-0.93) were associated with a significantly improved OS over observation (Figure 4B).

HER2 status is very an important predictor for antiHER2 treatment. Because only 120 HER2-positive patients remained after re-evaluating the HER2 status in the E2198 trial (46), we found no significant benefit for T-12 weeks versus observation in our sensitivity analysis (HR 0.78, 95\% CI: $0.57-1.07)$.

\section{Subgroup and sensitivity analyses}

Subgroup analysis showed that regardless of lymph node status in early breast cancer, T-12 months was associated with significantly improved DFS compared with observation. Shorter treatments (T-6 months and T-9 weeks) were not associated with a reduced DFS compared with T-12 months in patients with node-positive (HR 1.09, 95\% CI: 0.92-1.27, $\mathrm{I}^{2}=0.0 \%$ and HR $1.24,95 \%$ CI: $0.92-1.57, \mathrm{I}^{2}=39.3 \%$, respectively) or node-negative (HR 1.11, 95\% CI: 0.88-1.33, $\mathrm{I}^{2}=0.0 \%$ and HR $1.01,95 \%$ CI: $0.64-1.50, \mathrm{I}^{2}=59.2 \%$, respectively) early breast cancer (Figure S5).

Next, we performed subgroup analyses for hormone receptor status. T-12 months and T-24 months were associated with significantly improved DFS than observation in both hormone receptor-positive and hormone receptornegative early breast cancers. As for hormone receptor status, shorter treatments (T-6 months and T-9 weeks) were not associated with a significant reduction in DFS in patients with hormone-positive (HR 1.03, 95\% CI: 0.87$1.18, \mathrm{I}^{2}=0.0 \%$ and HR $1.23,95 \%$ CI: $0.94-1.52, \mathrm{I}^{2}=0.0 \%$, respectively) or hormone-negative (HR 1.15, 95\% CI: 0.96$1.34, \mathrm{I}^{2}=0.0 \%$ and HR $1.35,95 \%$ CI: $0.97-1.73, \mathrm{I}^{2}=35.2 \%$, respectively) early breast cancer when compared with T-12 months (Figure S6).

Based on the subgroup analysis by indirect comparisons, T-12 months and T-6 months were associated with significantly improved DFS than observation for lymph node-positive early breast cancer. No such association was observed between T-9 weeks and observation (HR 0.93, 95\% CI: 0.84-1.04) (Figure S7). However, T-12 months, T-6 months and T-9 weeks were associated with significantly improved DFS compared to observation for lymph node-negative early breast cancer (Figure S7). Additionally, the results of the sensitivity analyses after excluding the trials that respectively reported $1-3$ and $>3$ positive nodes remained essentially the same (Figure S7).

Subgroup and sensitivity analyses were also performed in hormone receptor-positive and hormone receptor-negative early breast cancer (Figure S8). T-24 months, T-12 months 
A

Author, year

12 months vs Observiation
Martine 2005
Romond 2005
Marc 2009
Slam on 2011
Slam on 2011
Perez 2011
Baselga 2012
Gianni 2014
Martine 2016
Gianni 2016
Subtotal

NMA

24 months vs Observiation

Martine 2005

Subtotal

NMA

9 weeks vs Observiation

Joensuu 2006

Subtotal

NMA

24 months vs 12 months

Martine 2005

Subtotal

NMA

NMA

months vs 12 months

Mavroudis 2015

Pivot 2019

Earl 2019

Subtotal

NMA

9 weeks vs 12 months

Conte 2017

Joensuu 2018

Subtotal

NMA

12 weeks vs 12 months

Schneider 2015

Subtotal

NMA

B Author, year

12 months vs Observation

Martine 2005

Martine 2005

Romond 2005

Marc 2009

Slam on 2011

Slam on 201

Perez 2011

Baselga 2012

Gianni 2014

Martine 201

Subtotal

24 months vs Observation

Martine 2005

Subtotal

NMA

9 weeks vs Observation

Joensuu 2006

Subtotal

NMA

24 months vs 12 months

Martine 2005

Subtotal

NMA

6 months vs 12 months

Mavroudis 2015

Pivot 2019

Earl 2019

Subtotal

9 weeks vs 12 months

Conte 2017

Joensuu 2018

Subtotal

Subto

12 weeks vs 12 months

Schneider 2015

Subtotal

NMA

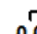

HR $(95 \% \mathrm{Cl})$ Weight (\%)

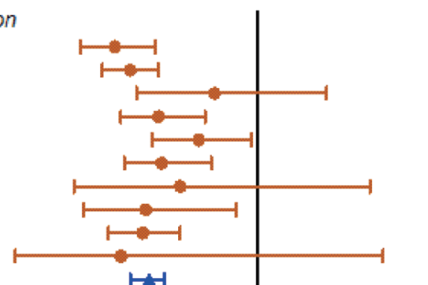

0.54(0.43-0.67) $\quad 15.49$

$0.59(0.50-0.68) \quad 22.31$

$0.86(0.61-1.22) \quad 3.22$

$0.68(0.56-0.83)^{*} \quad 13.06$

$0.81(0.66-0.98) \# \quad 10.04$

$0.69(0.57-0.85) \quad 12.37$

$0.75(0.41-1.36) \quad 1.38$

$0.64(0.44-0.93) \quad 4.82$

$0.63(0.52-0.75) \quad 16.42$

$0.65(0.59-0.70) \quad 100$

$0.83(0.81-0.86)$

$0.77(0.69-0.87) \quad 100$

$0.77(0.69-0.87) \quad 100$

$0.87(0.83-0.91)$

$0.42(0.21-0.83) \quad 100$

$0.42(0.21-0.83) \quad 100$

0.91(0.84-0.98)

$0.99(0.85-1.14) \quad 100$

$0.99(0.85-1.14) \quad 100$

1.03(0.99-1.08)

1.57(0.86-2.10) 4.44

$1.08(0.93-1.25) \quad 46.76$

$1.17(0.93-1.24) \quad 48.80$

$1.15(1.01-1.28) \quad 100$

1.06(1.02-1.08)

1.13(0.89-1.42) 46.19

$1.39(1.12-1.72) \quad 53.81$

$1.25(1.00-1.50) \quad 100$

1.08(1.01-1.16) ..

1.31(0.79-2.12) $\quad 100$

$1.13(0.90-1.40) \quad 100$

HR $(95 \% \mathrm{Cl}) \quad$ Weight (\%)

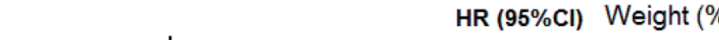

$0.76(0.65-0.88) \quad 28.10$ $0.66(0.54-0.79) \quad 24.29$ $1.27(0.68-2.38) \quad 0.59$ $0.62(0.48-0.79)^{\star} \quad 16.46$ $0.81(0.63-1.03) * 10.21$ $0.88(0.67-1.15) \quad 7.19$ $0.88(0.67-1.15) \quad 7.19$ $0.66(0.43-1.01) \quad 0.36$ $0.66(0.43-1.01) \quad 4.98$ $0.76(0.56-1.02) \quad 7.81$ $0.87(0.85-0.91) \quad 100$ $0.87(0.85-0.91)$ $0.74(0.63-0.86) \quad 100$ $0.74(0.63-0.86) \quad 100$ $0.88(0.84-0.93)$

$0.41(0.16-1.08) \quad 100$ $0.41(0.16-1.08) \quad 100$ $0.94(0.84-1.04)$

$0.94(0.84-1.04)$

1.05(0.86-1.28) $\quad 100$ $1.05(0.86-1.28) \quad 100$ $1.01(0.96-1.06)$

1.45(0.57-3.67) $\quad 1.01$ $\begin{array}{ll}1.14(0.92-1.39) & 43.95 \\ 1.14(0.95-1.37) & 55.04\end{array}$ $1.14(0.98-1.29) \quad 100$

$1.06(1.00-1.13)$

1.07(0.74-1.56) $\quad 55.19$

$\begin{array}{ll}1.36(0.98-1.89) & 44.19 \\ & 44.81\end{array}$

$\begin{array}{ll}1.36(0.98-1.89) & 44.81 \\ 1.20(0.90-1.50) & 100\end{array}$

$1.07(0.97-1.18)$

1.37(0.74-2.54) $\quad 100$ $1.15(0.87-1.51) \quad 100$

Figure 3 Pooled hazard ratios for disease-free survival (A) and overall survival (B) by network meta-analysis and pairwise meta-analysis. *, doxorubicin and cyclophosphamide, followed by docetaxel plus trastuzumab (AC-TH); ", docetaxel and carboplatin, given concurrently with trastuzumab, followed by trastuzumab (TCH). CI, confidence interval for pairwise meta-analysis and the credible interval for NMA; NMA, network meta-analysis. 


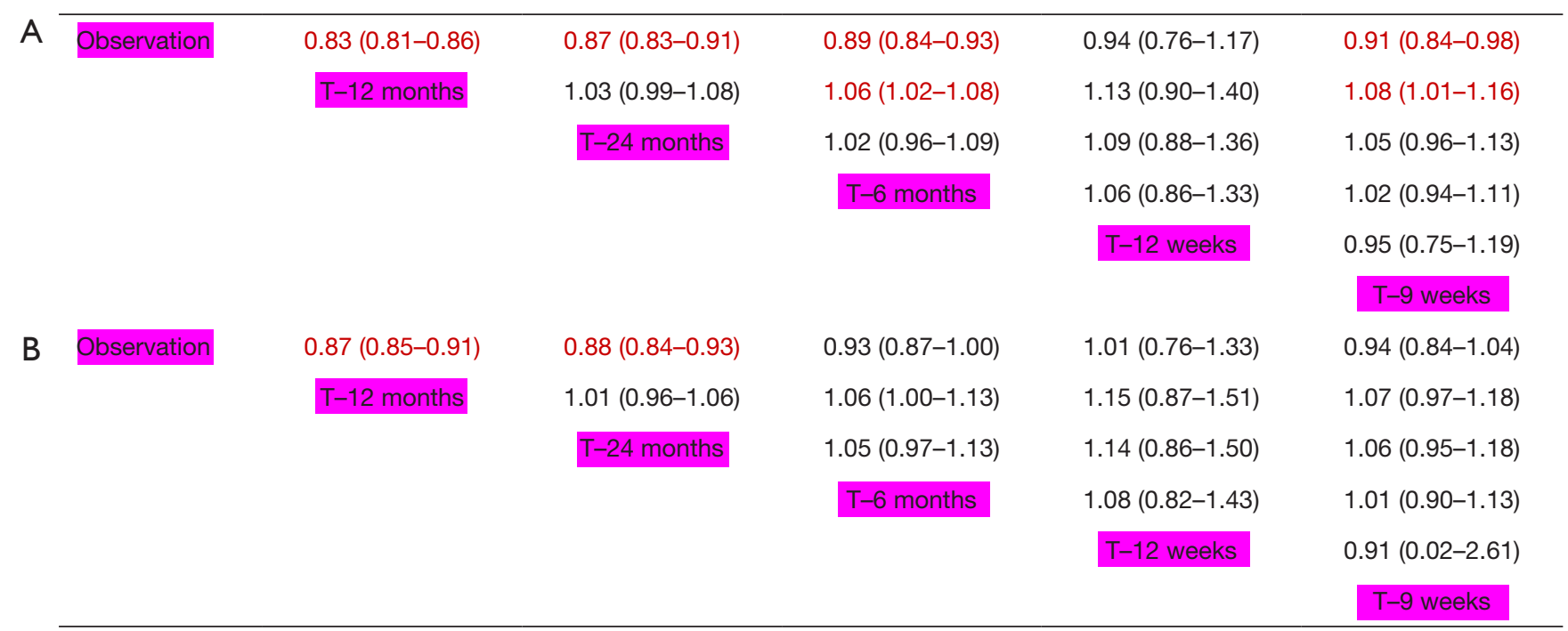

Figure 4 Pooled hazard ratios for disease-free survival (A) and overall survival (B). The columns were compared with the rows. Numbers in parentheses represent the $95 \%$ CI. HRs with $\mathrm{P}$ values of less than 0.05 were considered statistically significant (red). T, trastuzumab.

and T-6 months were associated with significantly improved DFS than observation regardless of the hormone receptor status. However, T-9 weeks might be better than T-12 months and T- 6 months. There were no differences among T-9 weeks, T-12 months, and T-6 months in the sensitivity analyses.

\section{Probability of efficacy and toxicity}

All 16 trials included in this NMA reported information about congestive heart failure. In addition, data on acceptability, asymptomatic decline in the left ventricular ejection fraction and grade 3-4 nonhematologic toxicities were available in 15, 13 and 14 studies, respectively. The SUCRA findings showed that the ranking for the optimum duration of trastuzumab treatments with the best average probability of PFS was as follows: T-12 months (95.6\%), T-24 months (69.6\%), T-6 months (53.2\%), T-9 weeks (41.2\%), T-12 weeks (34.3\%) and observation (6.1\%) (Figure 5A). Similar SUCRA results were found for OS; here, T-12 months was the most effective (86.7\%) treatment duration (Figure 5B).

T-24 months showed the worst effect in terms of elevated risk of trastuzumab-induced cardiotoxicity, with a mean probability of $83.6 \%$ for an asymptomatic decline in the left ventricular ejection fraction and $94.0 \%$ for congestive heart failure (Figure 5C,D). In addition, T-24 months conferred the greatest risk of grade 3-4 nonhematologic toxicities
(SUCRA: 95.8\%) (Figure 5E). Interestingly, T-12 months had the most frequent discontinued scheduled trastuzumab administration (SUCRA: 75\%) (Figure 5F).

\section{Risk-benefit analyses}

In our final analyses, we assessed the risk-benefit tradeoffs between the relative treatment effects (PFS and OS) and cardiotoxicity (congestive heart failure). Here, we used the SUCRA values to calculate the risk-benefit ratios. Then, based on the risk-benefit ratios, we could divide the duration of adjuvant trastuzumab treatments into four broad categories: (I) T-12 months and T-24 months; (II) T-6 months and T-12 weeks; (III) T-9 weeks; (IV) Observation. We found that both T-12 months and T-24 months were associated with significantly improved DFS and OS, but they both caused more congestive heart failure events than de-escalating treatments (Figure 6). In summary, T-12 months and T-24 months were more effective in terms of improving survival than shorter treatments but caused more cardiotoxicity.

\section{Discussion}

This NMA compared all major durations of adjuvant trastuzumab for HER2-positive early breast cancer and synthesized the available effects and safety of standard care, escalating and de-escalating treatments. Our results 
A

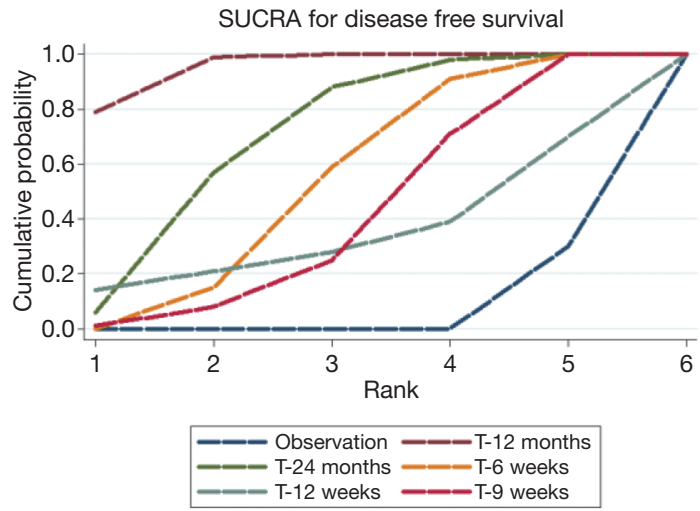

C

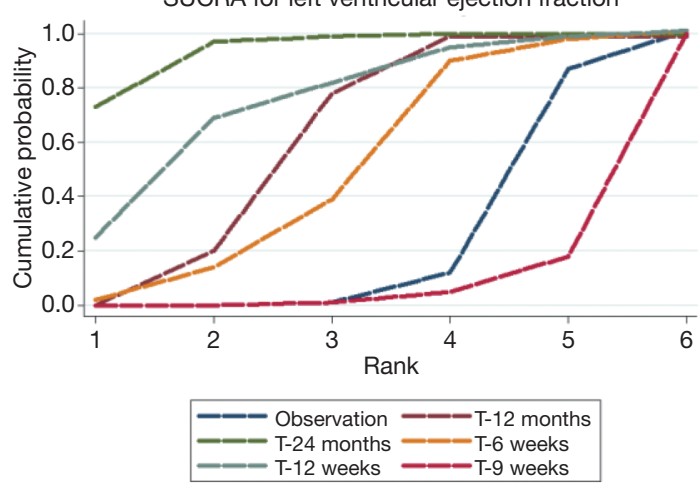

E

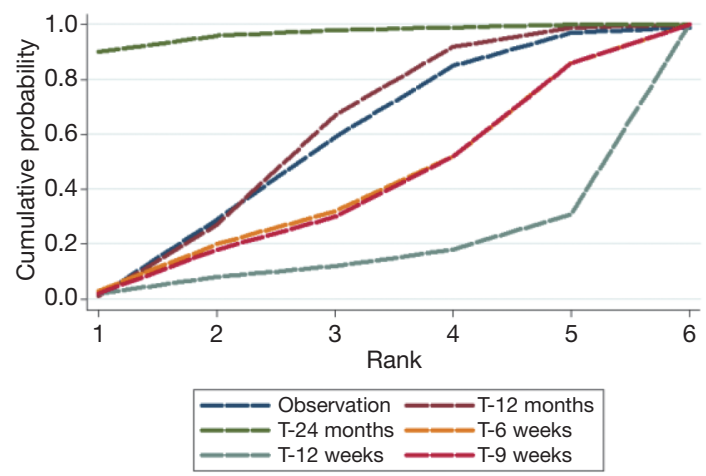

B

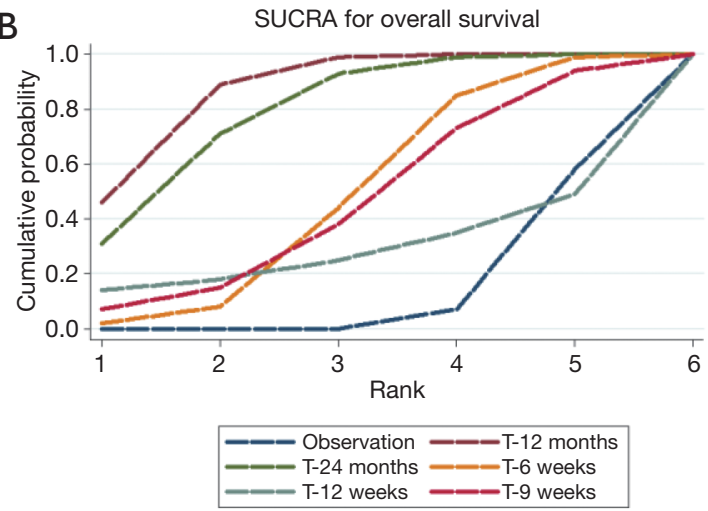

D

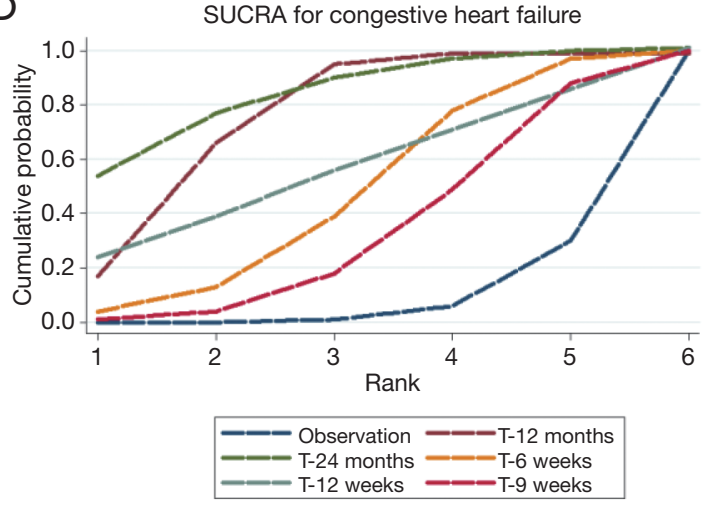

$\mathrm{F}$

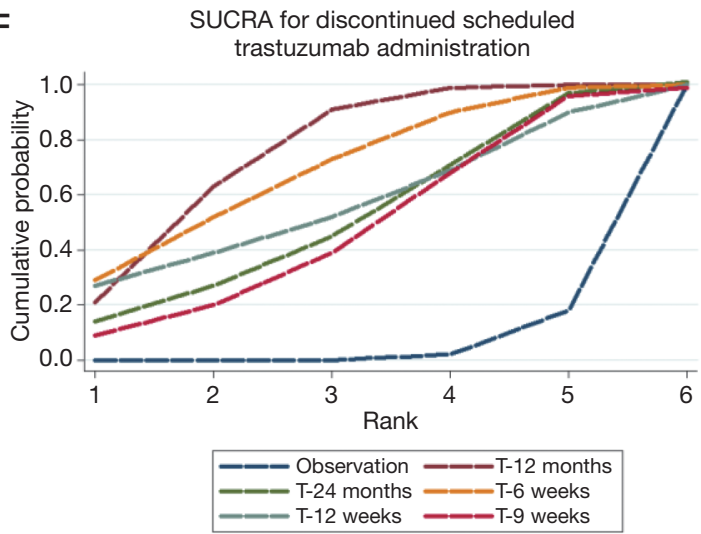

Figure 5 SUCRA values for the efficacy and trastuzumab tolerability in the NMA. SUCRA values for disease free survival (A), overall survival (B), left ventricular ejection fraction (C), congestive heart failure (D), grade 3-4 non-hematologic toxicities (E) and discontinued scheduled trastuzumab administration (F). The highest SUCRA value appeared at a depth of $100 \%$, and the lowest value was close to zero. SUCRA, surface under the cumulative ranking; T, trastuzumab.

confirm that trastuzumab treatment for 12 months (T12 months) is associated with a DFS and OS advantage over observation. Compared with T-12 months, escalating treatment (T-24 months) seems to provide no additional survival benefit but an increased risk of cardiac and grade 3-4 nonhematologic toxicities. T-6 months and T-9 weeks seem to provide a significant DFS advantage, as shown in the indirect comparisons to observation; additionally, T-6 months is associated with a significantly improved DFS compared to T-12 months. No such significance was found when comparing T-9 weeks and T-12 months in NMA. We found no benefit of de-escalating treatment was statistically 

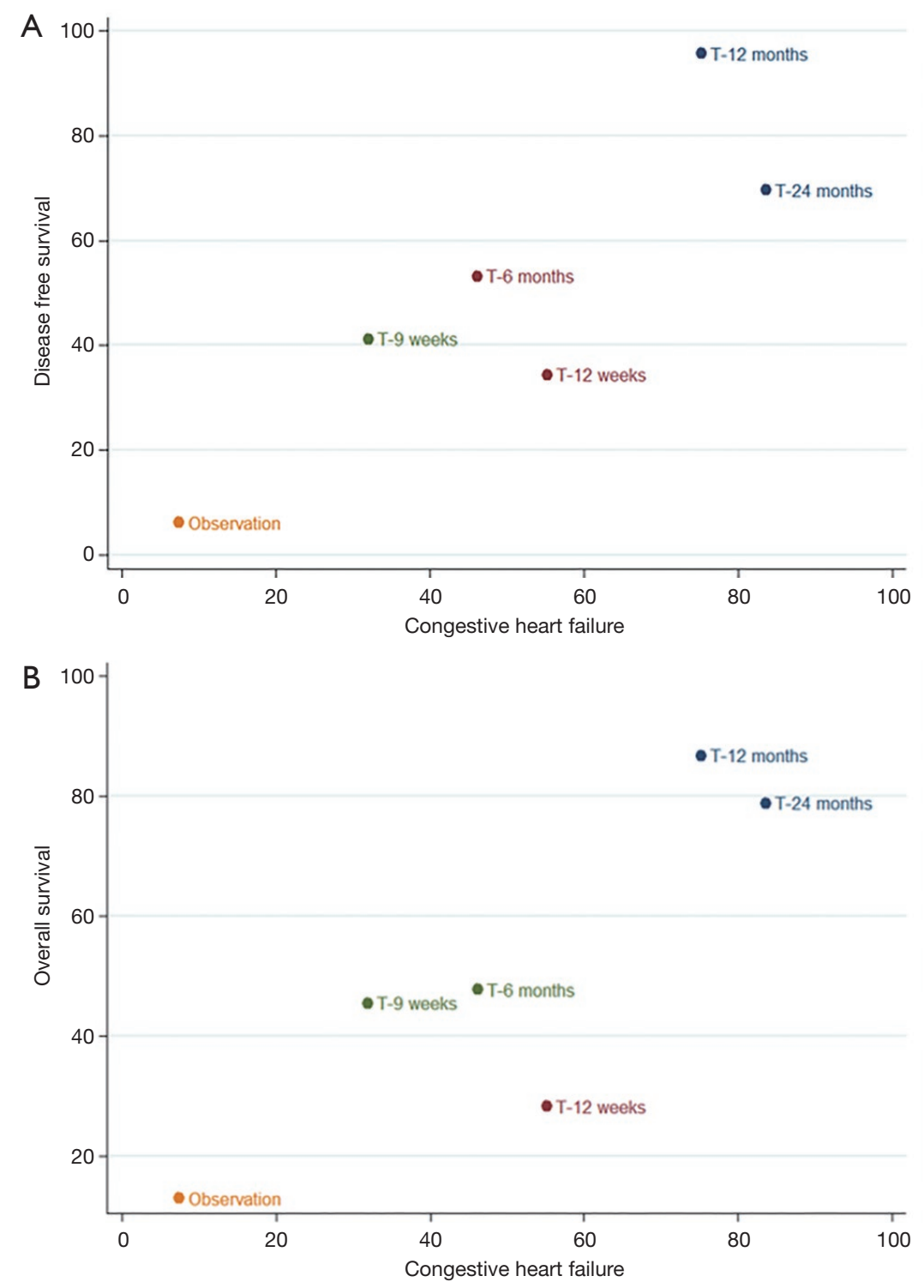

Figure 6 A risk-benefit analysis of congestive heart failure versus disease free survival or overall survival. SUCRA values for heart failure, disease free survival (A) or overall survival (B) were used to calculate the risk-benefit ratios in the NMA. The duration of adjuvant trastuzumab was divided into four broad categories according to the risk-benefit ratio. T, trastuzumab; SUCRA, surface under the cumulative ranking.

insignificant on OS in both the direct and indirect comparisons.

In agreement with our findings, earlier, traditional metaanalyses showed that 12 -month trastuzumab treatment improves DFS and OS in patients with HER2-positive early breast cancer but confers an increased risk of cardiotoxicity than shorter treatment protocols (12,14-17). Our study offered that both T-12 months and T-24 months were associated with significantly improved DFS and OS, but they both caused more congestive heart failure events than de-escalating treatments. The longer the use of trastuzumab, the higher the risk of trastuzumab induced cardiotoxicity. Selected patients, such as patients with a low risk of recurrence and with cardiac disease, might, therefore, be candidates for de-escalating treatments $(15,16)$. Previous studies also showed that T12-month trastuzumab treatment prevents disease recurrence and confers survival benefits for patients with HER2-positive early breast 
cancer compared with observation $(55,56)$. These previous meta-analyses, however, were limited to two durations of trastuzumab treatment: shorter (T-6 months, T-12 weeks, and T-9 weeks) or standard treatment. Our study thus adds to these meta-analyses by also assessing the effects of escalating treatment. In addition, we provide data on effectiveness and safety by making direct comparisons and indirect comparisons. We also grouped the de-escalating treatments into three categories, T-6 months, T-12 weeks, or T-9 weeks, and compared these durations with each by Bayesian analysis.

Our findings suggest that the benefits of standard care (T-12 months) compared to T-6 months (HR 1.15, 95\% CI: 1.01-1.28) and T-9 weeks were not associated with a DFS advantage in the direct comparisons (HR 1.25, 95\% CI: 1.00-1.50). By contrast, a previous sensitivity analysis in a traditional meta-analysis according to the duration of trastuzumab treatments found a trend favoring T-12 months compared to both T-6 months (HR 1.18, 95\% CI: 1.00 1.39) and T-9 weeks (HR 1.21, 95\% CI: 1.06-1.53) (12). These results are inconsistent with those of our direct comparisons, due in part to the updated data included in our study. Our indirect results suggest that T-12 months provides a significant DFS advantage compared to either T-6 months (HR 1.06, 95\% CI: 1.02-1.08) or T-9 weeks (HR 1.08, 95\% CI: 1.01-1.16) in patients with HER2positive early breast cancer. We also found by indirect analysis that T-6 months (HR 1.06, 95\% CI: 1.02-1.08) and T-9 weeks (HR 1.08, 95\% CI: 1.01-1.16) seem to be better DFS than observation. Based on these findings, deescalating treatments might be a new choice for HER2positive early breast cancer.

Our study is the first NMA, to the best of our knowledge, to compare the DFS of six interventions in subgroup and sensitivity analyses according to the lymph node and hormone status and among patients who received adjuvant and neoadjuvant treatments. The findings of our pairwise meta-analyses were consistent with the NMA, regardless of lymph node positivity or negativity. The pooled analysis suggests that T-12 months treatment confers a significant benefit in terms of DFS compared to observation, while we detected no significant benefit for de-escalating or standard treatments in the subgroup analysis. We also found that patients with lymph node negativity showed notable benefits from T-6 months and T-9 weeks treatment. A sensitivity analysis, however, revealed that there was a trend favoring T-9 weeks treatment based on the number of positive lymph nodes ( $\geq 1,1-3$ and $\geq 4$ positive nodes). These data suggest that lymph node status might not be a biomarker for deescalating treatments.

For hormone receptor status, we similarly found that T-12 months and T-24 months were associated with increased DFS compared with observation, regardless of the hormone receptor status. In addition, T-6 months and T-9 weeks showed significant benefits of DFS compared to observation in the hormone receptor-positive and hormone receptor-negative subgroup. By contrast, T-9 weeks provided a DFS advantage in hormone receptornegative patients according to the subgroup analysis compared to T-12 months, but not be confirmed in the sensitivity analysis. The optimum duration of trastuzumab treatment in patients with HER2-positive early breast cancer is currently unknown. These data suggest, however, that even if the standard treatment time is not achievable (T-12 months), it is better to use adjuvant trastuzumab than to perform observation. De-escalating treatments might also be an appropriate treatment option in selected patients, such as hormone receptor- negative patients. However, our results need to be confirmed with additional studies.

Some limitations of our NMA should be noted. Our review did not clarify the role of chemotherapy, lapatinib or pertuzumab for all trastuzumab treatment durations. However, a previous NMA detected a statistically significant difference in all approved trastuzumab-containing chemotherapies (57). This finding implies that clinical use of chemotherapy regimens combined with different trastuzumab durations show different benefits and harms for early-stage HER2-positive breast cancer. Future work should, therefore, combine chemotherapy protocols with anti-HER2 treatment times in the NMA. Furthermore, we also included patients with neoadjuvant trastuzumabcontaining chemotherapy, whose clinical efficacy might be affected by tumor stage, as most patients had locally advanced breast cancer. In our study, we found that lymph node status might not be a good marker for de-escalating treatments in subgroup analysis based on lymph node status. Finally, this NMA had some missing patient information in the published data; thus, the cardiac events and grade 3-4 nonhematologic toxicities might be lower than those in clinical practice and might have affected the rank and riskbenefit assessment of efficacy and toxicity.

\section{Conclusions}

In conclusion, the results of our NMA clearly show that T-12 months is the best standard-of-care for HER2-positive 
early breast cancers. The unanswered question arises as to identify low risk patients with HER2-positive early breast cancer suited for de-escalating regimens of chemotherapy with trastuzumab durations. Importantly, our data support that if treatment does not reach the standard treatment time, it is better to use adjuvant trastuzumab than to perform observation. While de-escalating treatments confer only a small improvement in DFS in indirect comparisons, shorter treatment durations might be an appropriate choice for patients with hormone receptor- negative.

\section{Acknowledgments}

Funding: The network meta-analysis was supported by the National Natural Science Foundation of China (Grant No.81773097) and Program of Chengdu Science and Technology Bureau (Grant No. 2018-YF05-01326-SN).

\section{Footnote}

Reporting Checklist: The authors have completed the PRISMA reporting checklist. Available at http://dx.doi. org/10.21037/tcr-20-2378

Conflicts of Interest: All authors have completed the ICMJE uniform disclosure form (available at http://dx.doi. org/10.21037/tcr-20-2378). The authors have no conflicts of interest to declare.

Ethical Statement: The authors are accountable for all aspects of the work in ensuring that questions related to the accuracy or integrity of any part of the work are appropriately investigated and resolved.

Open Access Statement: This is an Open Access article distributed in accordance with the Creative Commons Attribution-NonCommercial-NoDerivs 4.0 International License (CC BY-NC-ND 4.0), which permits the noncommercial replication and distribution of the article with the strict proviso that no changes or edits are made and the original work is properly cited (including links to both the formal publication through the relevant DOI and the license). See: https://creativecommons.org/licenses/by-nc-nd/4.0/.

\section{References}

1. Slamon DJ, Clark GM, Wong SG, et al. Human breast cancer: correlation of relapse and survival with amplification of the HER-2/neu oncogene. Science 1987;235:177-82.

2. Slamon DJ, Godolphin W, Jones LA, et al. Studies of the HER-2/neu proto-oncogene in human breast and ovarian cancer. Science 1989;244:707-12.

3. Curigliano G, Viale G, Bagnardi V, et al. Clinical relevance of HER2 overexpression/amplification in patients with small tumor size and node-negative breast cancer. J Clin Oncol 2009;27:5693-9.

4. Piccart-Gebhart MJ, Procter M, Leyland-Jones B, et al. Trastuzumab after adjuvant chemotherapy in HER2positive breast cancer. N Engl J Med 2005;353:1659-72.

5. Hortobagyi GN. Trastuzumab in the treatment of breast cancer. N Engl J Med 2005;353:1734-6.

6. Romond EH, Perez EA, Bryant J, et al. Trastuzumab plus adjuvant chemotherapy for operable HER2-positive breast cancer. N Engl J Med 2005;353:1673-84.

7. Slamon D, Eiermann W, Robert N, et al. Adjuvant trastuzumab in HER2-positive breast cancer. N Engl J Med 2011;365:1273-83.

8. Coates AS, Winer EP, Goldhirsch A, et al. Tailoring therapies--improving the management of early breast cancer: St Gallen International Expert Consensus on the Primary Therapy of Early Breast Cancer 2015. Ann Oncol 2015;26:1533-46.

9. Curigliano G, Burstein HJ, Winer EP, et al. De-escalating and escalating treatments for early-stage breast cancer: the St. Gallen International Expert Consensus Conference on the Primary Therapy of Early Breast Cancer 2017. Ann Oncol 2017;28:1700-12.

10. Goetz MP, Gradishar WJ, Anderson BO, et al. NCCN Guidelines Insights: Breast Cancer, Version 3.2018. J Natl Compr Canc Netw 2019;17:118-26.

11. Stewart P, Blanchette P, Shah PS, et al. Do all patients with HER2 positive breast cancer require one year of adjuvant trastuzumab? A systematic review and meta-analysis. Breast 2020;54:203-10.

12. Niraula S, Gyawali B. Optimal duration of adjuvant trastuzumab in treatment of early breast cancer: a metaanalysis of randomized controlled trials. Breast Cancer Res Treat 2019;173:103-9.

13. Earl HM, Hiller L, Vallier AL, et al. 6 versus 12 months of adjuvant trastuzumab for HER2-positive early breast cancer (PERSEPHONE): 4-year disease-free survival results of a randomised phase 3 non-inferiority trial. Lancet 2019;393:2599-612.

14. Gyawali B, Niraula S. Duration of adjuvant trastuzumab in HER2 positive breast cancer: Overall and disease 
free survival results from meta-analyses of randomized controlled trials. Cancer Treat Rev 2017;60:18-23.

15. Chen $\mathrm{L}, \mathrm{Zhou} \mathrm{W}, \mathrm{Hu} \mathrm{X}$, et al. Short-duration versus 1-year adjuvant trastuzumab in early HER2 positive breast cancer: A meta-analysis of randomized controlled trials. Cancer Treat Rev 2019;75:12-9.

16. Inno A, Barni S, Ghidini A, et al. One year versus a shorter duration of adjuvant trastuzumab for HER2-positive early breast cancer: a systematic review and meta-analysis. Breast Cancer Res Treat 2019;173:247-54.

17. Goldvaser H, Korzets Y, Shepshelovich D, et al. Deescalating Adjuvant Trastuzumab in HER2-Positive Early-Stage Breast Cancer: A Systemic Review and MetaAnalysis. JNCI Cancer Spectr 2019;3:pkz033.

18. Rouse B, Chaimani A, Li T. Network meta-analysis: an introduction for clinicians. Intern Emerg Med 2017;12:103-11.

19. Goldhirsch A, Gelber RD, Piccart-Gebhart MJ, et al. 2 years versus 1 year of adjuvant trastuzumab for HER2positive breast cancer (HERA): an open-label, randomised controlled trial. Lancet 2013;382:1021-8.

20. Liberati A, Altman DG, Tetzlaff J, et al. The PRISMA statement for reporting systematic reviews and metaanalyses of studies that evaluate healthcare interventions: explanation and elaboration. BMJ 2009;339:b2700.

21. Hutton B, Salanti G, Caldwell DM, et al. The PRISMA extension statement for reporting of systematic reviews incorporating network meta-analyses of health care interventions: checklist and explanations. Ann Intern Med 2015;162:777-84.

22. Hu Q, Wang X, Chen Y, et al. Optimum duration of adjuvant trastuzumab in treatment of human epidermal growth factor receptor-2 positive early breast cancer: protocol for a network meta-analysis of randomised controlled trials. BMJ Open 2020;10:e035802.

23. Russell SD, Blackwell KL, Lawrence J, et al. Independent adjudication of symptomatic heart failure with the use of doxorubicin and cyclophosphamide followed by trastuzumab adjuvant therapy: a combined review of cardiac data from the National Surgical Adjuvant breast and Bowel Project B-31 and the North Central Cancer Treatment Group N9831 clinical trials. J Clin Oncol 2010;28:3416-21.

24. Yu AF, Singh JC, Wang R, et al. Cardiac Safety of Dual Anti-HER2 Therapy in the Neoadjuvant Setting for Treatment of HER2-Positive Breast Cancer. Oncologist 2017;22:642-7.
25. Salanti G. Indirect and mixed-treatment comparison, network, or multiple-treatments meta-analysis: many names, many benefits, many concerns for the next generation evidence synthesis tool. Res Synth Methods 2012;3:80-97.

26. Mavridis D, Giannatsi M, Cipriani A, et al. A primer on network meta-analysis with emphasis on mental health. Evid Based Ment Health 2015;18:40-6.

27. Higgins JP, Altman DG, Gotzsche PC, et al. The Cochrane Collaboration's tool for assessing risk of bias in randomised trials. BMJ 2011;343:d5928.

28. Salanti G, Del Giovane C, Chaimani A, et al. Evaluating the quality of evidence from a network meta-analysis. PLoS One 2014;9:e99682.

29. Guyatt GH, Oxman AD, Vist GE, et al. GRADE: an emerging consensus on rating quality of evidence and strength of recommendations. BMJ 2008;336:924-6.

30. Chaimani A, Higgins JP, Mavridis D, et al. Graphical tools for network meta-analysis in STATA. PLoS One 2013;8:e76654.

31. Song F, Freemantle N, Sheldon TA, et al. Selective serotonin reuptake inhibitors: meta-analysis of efficacy and acceptability. BMJ 1993;306:683-7.

32. Tierney JF, Stewart LA, Ghersi D, et al. Practical methods for incorporating summary time-to-event data into metaanalysis. Trials 2007;8:16.

33. Woods BS, Hawkins N, Scott DA. Network meta-analysis on the log-hazard scale, combining count and hazard ratio statistics accounting for multi-arm trials: a tutorial. BMC Med Res Methodol 2010;10:54.

34. Veroniki AA, Vasiliadis HS, Higgins JP, et al. Evaluation of inconsistency in networks of interventions. Int J Epidemiol 2013;42:332-45.

35. Dias S, Welton NJ, Sutton AJ, et al. Evidence synthesis for decision making 4: inconsistency in networks of evidence based on randomized controlled trials. Med Decis Making 2013;33:641-56.

36. Brooks SP, Gelman A. General methods for monitoring convergence of iterative simulations. Journal of Computational and Graphical Statistics 1998;7:434-55.

37. Higgins JP, Thompson SG, Deeks JJ, et al. Measuring inconsistency in meta-analyses. BMJ 2003;327:557-60.

38. Pivot X, Romieu G, Debled M, et al. 6 months versus 12 months of adjuvant trastuzumab for patients with HER2positive early breast cancer (PHARE): a randomised phase 3 trial. Lancet Oncol 2013;14:741-8.

39. Joensuu H, Bono P, Kataja V, et al. Fluorouracil, 
epirubicin, and cyclophosphamide with either docetaxel or vinorelbine, with or without trastuzumab, as adjuvant treatments of breast cancer: final results of the FinHer Trial. J Clin Oncol 2009;27:5685-92.

40. Joensuu H, Fraser J, Wildiers H, et al. Effect of Adjuvant Trastuzumab for a Duration of 9 Weeks vs 1 Year With Concomitant Chemotherapy for Early Human Epidermal Growth Factor Receptor 2-Positive Breast Cancer: The SOLD Randomized Clinical Trial. JAMA Oncol 2018;4:1199-206.

41. Mavroudis D, Saloustros E, Malamos N, et al. Six versus 12 months of adjuvant trastuzumab in combination with dose-dense chemotherapy for women with HER2-positive breast cancer: a multicenter randomized study by the Hellenic Oncology Research Group (HORG). Ann Oncol 2015;26:1333-40.

42. Perez EA, Romond EH, Suman VJ, et al. Trastuzumab plus adjuvant chemotherapy for human epidermal growth factor receptor 2-positive breast cancer: planned joint analysis of overall survival from NSABP B-31 and NCCTG N9831. J Clin Oncol 2014;32:3744-52.

43. Piccart-Gebhart M, Holmes E, Baselga J, et al. Adjuvant Lapatinib and Trastuzumab for Early Human Epidermal Growth Factor Receptor 2-Positive Breast Cancer: Results From the Randomized Phase III Adjuvant Lapatinib and/ or Trastuzumab Treatment Optimization Trial. J Clin Oncol 2016;34:1034-42.

44. Schneider BP, O'Neill A, Shen F, et al. Pilot trial of paclitaxel-trastuzumab adjuvant therapy for early stage breast cancer: a trial of the ECOG-ACRIN cancer research group (E2198). Br J Cancer 2015;113:1651-7.

45. Spielmann M, Roche H, Delozier T, et al. Trastuzumab for patients with axillary-node-positive breast cancer: results of the FNCLCC-PACS 04 trial. J Clin Oncol 2009;27:6129-34.

46. de Azambuja E, Holmes AP, Piccart-Gebhart M, et al. Lapatinib with trastuzumab for HER2-positive early breast cancer (NeoALTTO): survival outcomes of a randomised, open-label, multicentre, phase 3 trial and their association with pathological complete response. Lancet Oncol 2014;15:1137-46.

47. Gianni L, Pienkowski T, Im YH, et al. 5-year analysis of neoadjuvant pertuzumab and trastuzumab in patients with locally advanced, inflammatory, or early-stage HER2-positive breast cancer (NeoSphere): a multicentre, open-label, phase 2 randomised trial. Lancet Oncol 2016;17:791-800.
48. Gianni L, Eiermann W, Semiglazov V, et al. Neoadjuvant and adjuvant trastuzumab in patients with HER2-positive locally advanced breast cancer (NOAH): follow-up of a randomised controlled superiority trial with a parallel HER2-negative cohort. Lancet Oncol 2014;15:640-7.

49. Pivot X, Romieu G, Debled M, et al. 6 months versus 12 months of adjuvant trastuzumab in early breast cancer (PHARE): final analysis of a multicentre, open-label, phase 3 randomised trial. Lancet 2019;393:2591-8.

50. Joensuu H, Kellokumpu-Lehtinen PL, Bono P, et al. Adjuvant docetaxel or vinorelbine with or without trastuzumab for breast cancer. N Engl J Med 2006;354:809-20.

51. Conte P, Frassoldati A, Bisagni G, et al. Nine weeks versus 1 year adjuvant trastuzumab in combination with chemotherapy: final results of the phase III randomized Short-HER studydouble dagger. Ann Oncol 2018;29:2328-33.

52. Ganz PA, Romond EH, Cecchini RS, et al. Long-Term Follow-Up of Cardiac Function and Quality of Life for Patients in NSABP Protocol B-31/NRG Oncology: A Randomized Trial Comparing the Safety and Efficacy of Doxorubicin and Cyclophosphamide (AC) Followed by Paclitaxel With AC Followed by Paclitaxel and Trastuzumab in Patients With Node-Positive Breast Cancer With Tumors Overexpressing Human Epidermal Growth Factor Receptor 2. J Clin Oncol 2017;35:3942-8.

53. Romond EH, Jeong JH, Rastogi P, et al. Seven-year follow-up assessment of cardiac function in NSABP B-31, a randomized trial comparing doxorubicin and cyclophosphamide followed by paclitaxel (ACP) with ACP plus trastuzumab as adjuvant therapy for patients with node-positive, human epidermal growth factor receptor 2-positive breast cancer. J Clin Oncol 2012;30:3792-9.

54. Gianni L, Dafni U, Gelber RD, et al. Treatment with trastuzumab for 1 year after adjuvant chemotherapy in patients with HER2-positive early breast cancer: a 4-year follow-up of a randomised controlled trial. Lancet Oncol 2011;12:236-44.

55. Viani GA, Afonso SL, Stefano EJ, et al. Adjuvant trastuzumab in the treatment of her-2-positive early breast cancer: a meta-analysis of published randomized trials. BMC Cancer 2007;7:153.

56. Genuino AJ, Chaikledkaew U, The DO, et al. Adjuvant trastuzumab regimen for HER2-positive early-stage breast cancer: a systematic review and meta-analysis. Expert Rev 
Clin Pharmacol 2019;12:815-24.

57. Shen Y, Fujii T, Ueno NT, et al. Comparative efficacy of adjuvant trastuzumab-containing chemotherapies

Cite this article as: Ma J, Tang $\mathrm{X}, \mathrm{Hu} \mathrm{Q}$, Wang Q, Chen Y, Li X, Luo T, Cao D. Optimum adjuvant trastuzumab duration for human epidermal growth factor receptor-2 positive breast cancer: a network meta-analysis of randomized trials. Transl Cancer Res 2021;10(4):1628-1643. doi: 10.21037/tcr-20-2378 for patients with early HER2-positive primary breast cancer: a network meta-analysis. Breast Cancer Res Treat 2019;173:1-9. 


\section{Supplementary}

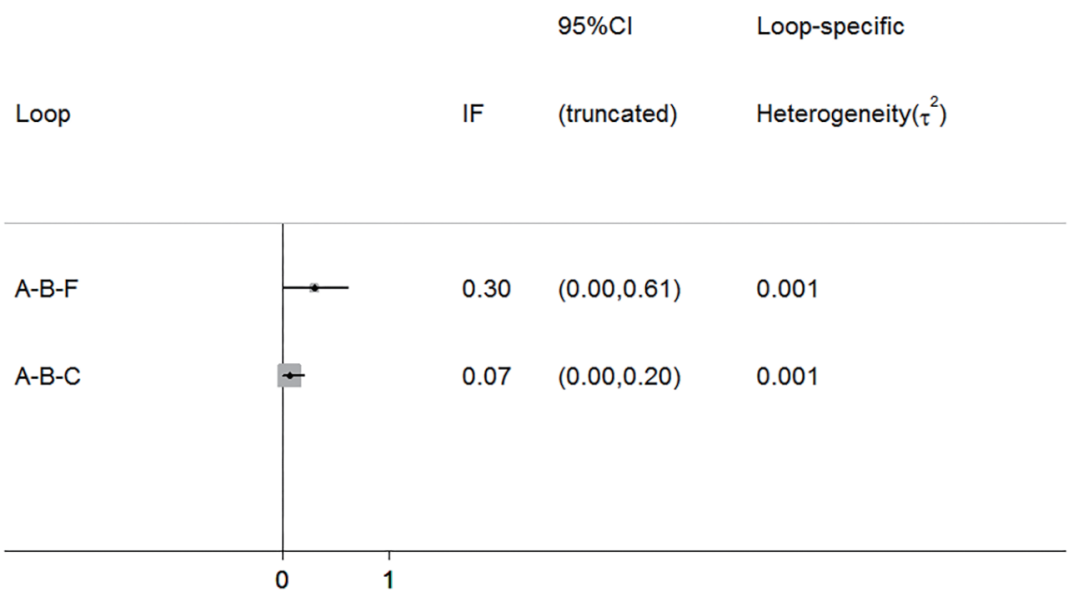

Figure S1 Inconsistency plot for the optimal duration of adjuvant trastuzumab network. Two triangular loops were found in the six comparisons. The $\mathrm{P}_{(\mathrm{A}-\mathrm{B}-\mathrm{F})}$ was 0.067 , and the $\mathrm{P}_{(\mathrm{A}-\mathrm{B}-\mathrm{C})}$ was 0.321 . A, observation; B, T-12 months; C, T-24 months; F, T-9 weeks; and T, trastuzumab.

\section{Contribution plot for the optimal duration}

Direct comparisons in the network

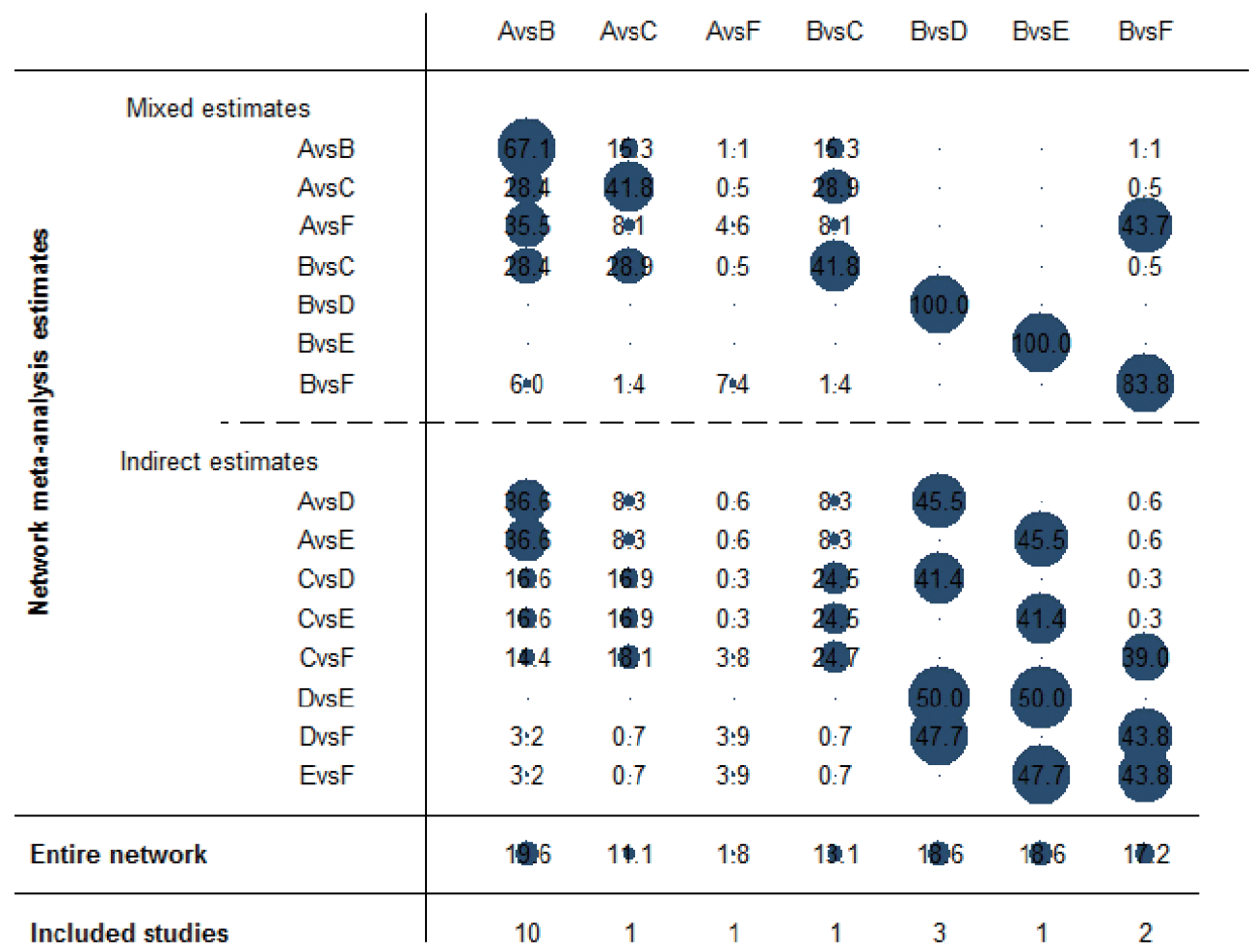

Figure S2 Contribution plot for the optimal duration of adjuvant trastuzumab. The numbers represent the weights as percentages (\%). The size of each circle is proportional to the weights of the direct comparisons (horizontal axis). A, observation; B, T-12 months; C, T-24 months; D, T-6 months; E, T-12 weeks; F, T-9 weeks; and T, trastuzumab. 
Random sequence generation (selection bias)

Allocation concealment (selection bias)

Blinding of participants and personnel (performance bias)

Blinding of outcome assessment (detection bias)

incomplete outcome data (attrition bias)

Selective reporting (reporting bias)

Other bias

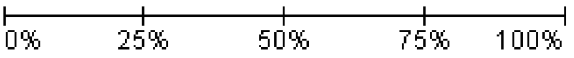

Low risk of bias

Unclear risk of bias $\square$ High risk of bias
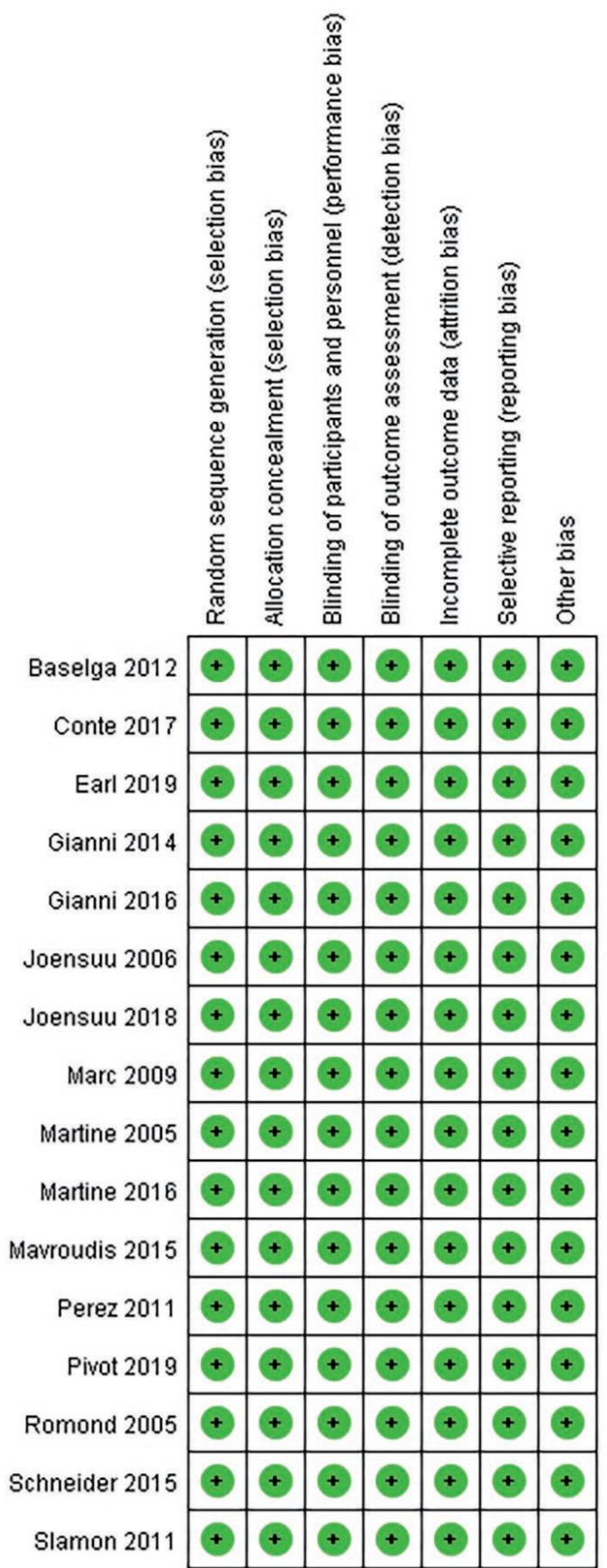

Figure S3 Risk of bias summary of the RCTs included in the network meta-analysis. RCTs, randomized controlled trials. 


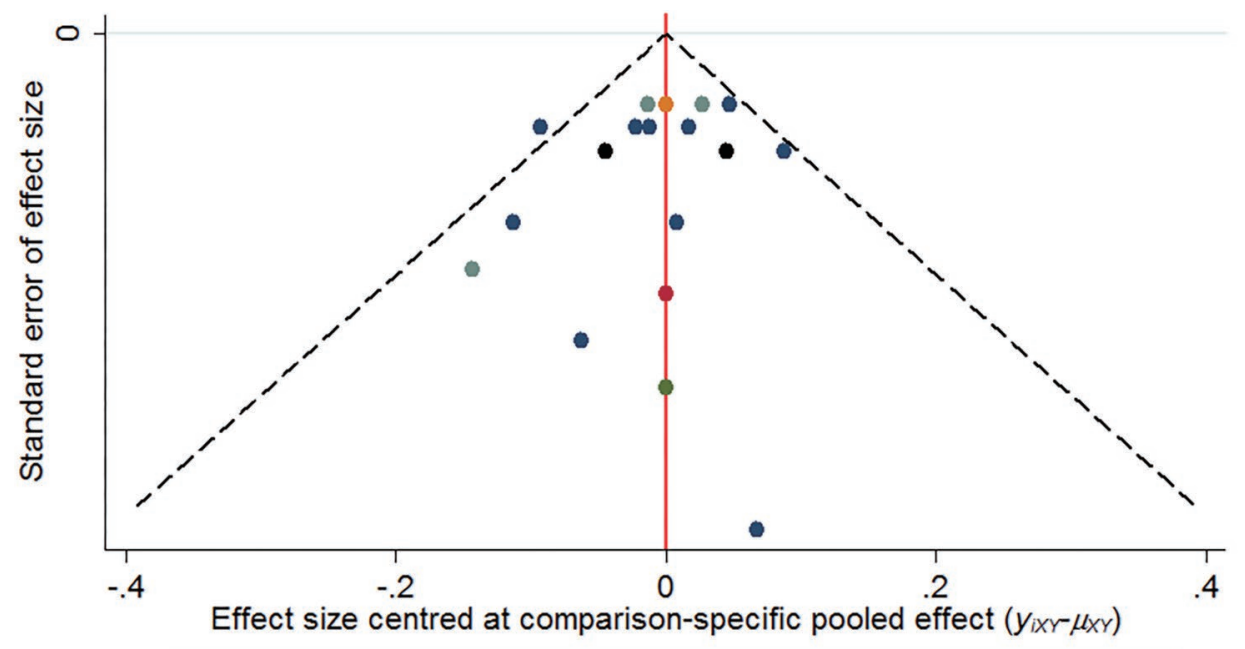

- Observation vs T-12months Observation vs T-24 months

- Observation vs T-9 weeks T-12 months vs T-24 months

- T-12 months vs T-24 months - T-12 months vs T-12 weeks

- T-12 months vs T-9 weeks

Figure S4 Comparison-adjusted funnel plot for the optimum duration of adjuvant trastuzumab. T, trastuzumab.

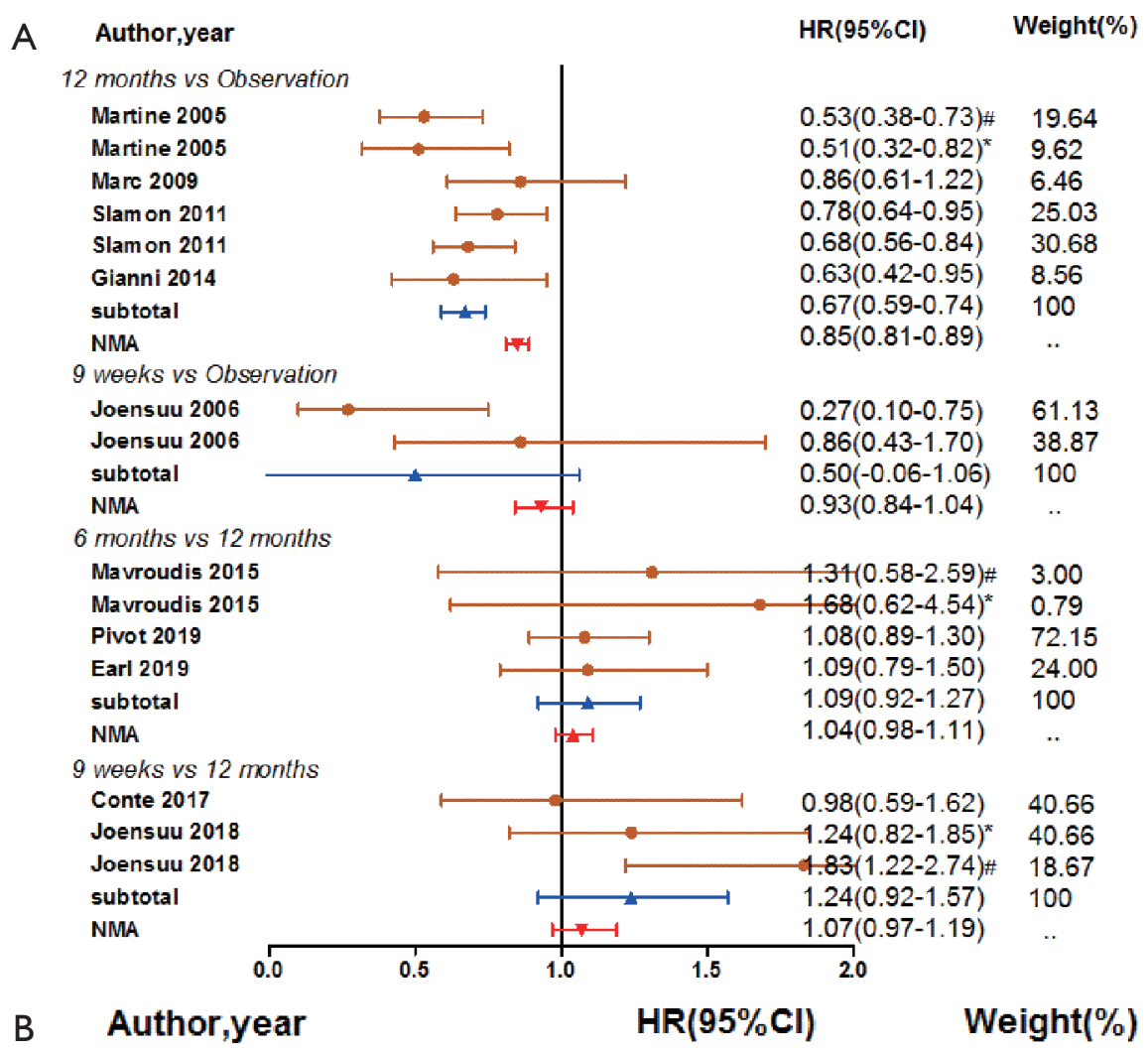

Buthor,year

$\mathrm{HR}(95 \% \mathrm{Cl})$

Weight(\%)

12 months vs Observation

\begin{tabular}{|c|c|c|c|c|}
\hline Martine 2005 & $\mapsto-1$ & & $0.51(0.30-0.87)$ & 29.16 \\
\hline Slam on 2011 & $\mapsto$ & & $0.47(0.28-0.77)$ & 39.47 \\
\hline Slam on 2011 & $1 \bullet-1$ & & $0.64(0.41-1.01)$ & 26.32 \\
\hline Gianni 2014 & $\longmapsto$ & $\longrightarrow$ & $0.60(0.23-1.60)$ & 5.05 \\
\hline Subtotal & $\mapsto H$ & & $0.53(0.38-0.69)$ & 100 \\
\hline NMA & $m$ & & $0.77(0.69-0.89)$ & ." \\
\hline \multicolumn{5}{|c|}{6 months vs 12 months } \\
\hline Ma vroud is 2015 & & & $3.78(0.39-36.48)$ & 0.02 \\
\hline Pivot 2019 & 5 & $4-1$ & $1.08(0.85-1.37)$ & 73.67 \\
\hline Earl 2019 & $\mapsto$ & $\rightarrow-\rightarrow$ & $1.18(0.82-1.69)$ & 26.32 \\
\hline Subtotal & & +1 & $1.11(0.88-1.33)$ & 100 \\
\hline NMA & & 4 & $1.05(0.96-1.14)$ & ". \\
\hline \multicolumn{5}{|c|}{9 weeks vs 12 months } \\
\hline Conte 2017 & $\mapsto$ & $\rightarrow$ & $0.87(0.59-1.29)$ & 53.91 \\
\hline Joensuu 2018 & & $\longrightarrow \longrightarrow$ & $1.31(0.95-1.80)$ & 46.09 \\
\hline Subtotal & $\longmapsto$ & $\longrightarrow$ & $1.07(0.64-1.50)$ & 100 \\
\hline NMA & & $H$ & $1.05(0.96-1.17)$ & ." \\
\hline & & & $\begin{array}{ll}1 & 1 \\
3 & 4\end{array}$ & \\
\hline
\end{tabular}

Figure S5 Subgroup analysis for disease-free survival based on the lymph node status. The pooled hazard ratios for lymph node-positive (A) and lymph node-negative patients (B) were produced by network meta-analysis and pairwise meta-analysis. *, 1-3 lymph nodes positive; \#, $\geq 4$ lymph nodes positive. CI, confidence interval for pairwise meta-analysis and the credible interval for network meta-analysis; NMA, network meta-analysis. 
A Author,year

12 months vs Observation

Martine 2005

Martine 2005

Martine 2005

Slam on 2011

Slam on 2011

Edith 2014

Gianni 2014

subtotal

NMA

24 months vs Observation

Martine 2005

subtotal

NMA

6 months vs 12 months

Mavroudis 2015

Mavroudis 2015

Pivot 2019

Earl 2019

subtotal

NMA

9 weeks vs 12 months

Conte 2017

Joensuu 2018

subtotal

NMA

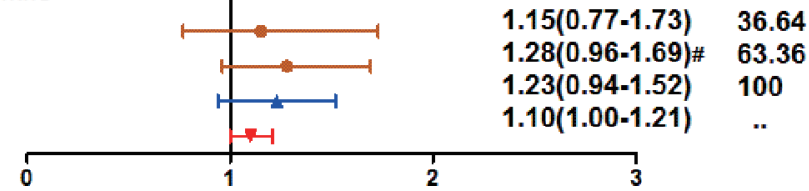

B Author,year

12 months vs Observation

Martine 2005

Slam on 2011

Slam on 2011

Edith 2014

Gianni 2014

Subtatol

NMA

24 months vs Observation

Martine 2005

Subtotal

NMA

24 months vs 12 months

Martine 2005

Subtotal

NMA

6 months vs 12 months

Mavroudis 2015

Mavroudis 2015

Pivot 2019

Earl 2019

Subtatol

NMA

9 weeks vs 12 months

Conte 2017

Joensuu 2018

Subtatol

NMA

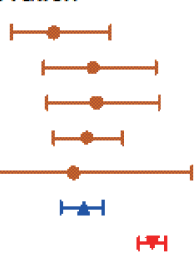

$\longmapsto$

$\longmapsto$

$\mapsto-1$

ths

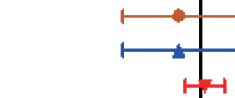

ths

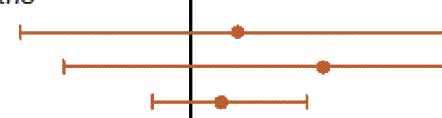

$0.52(0.39-0.69)$

$0.64(0.49-0.83)$

$0.65(0.50-0.84)$

$0.62(0.52-0.73)$

$0.58(0.35-0.94)$

0.61(0.54-0.67)

$0.82(0.78-0.86)$

$0.70(0.59-0.83)$

$0.70(0.59-0.83)$

$0.83(0.78-0.88)$

$0.93(0.76-1.14)$

$0.93(0.76-1.14)$

1.01(0.95-1.07)

$1.44(0.48-2.69)^{*}$

(0.61-3.20)* 2.10

$1.09(0.88-1.35) \quad 63.68$

1.26(0.97-1.64) 31.34

$1.15(0.96-1.34) \quad 100$

1.07(1.00-1.15) ..

6.27

53.73

100

..

Figure S6 Subgroup analysis based on hormone receptor status. The pooled hazard ratios for hormone receptor-positive (A) and hormone receptor-negative patients (B) produced by network meta-analysis and pairwise meta-analysis. * , estrogen receptor positive; *, progesteroneor estrogen-receptor negative. CI, confidence interval for the pairwise meta-analysis and the credible interval for network meta-analysis; NMA, network meta-analysis. 


\begin{tabular}{|c|c|c|c|c|}
\hline \multirow[t]{4}{*}{ A } & Observation & $0.85(0.81-0.89)$ & $0.88(0.81-0.96)$ & $0.93(0.84-1.04)$ \\
\hline & & T-12 months & $1.04(0.98-1.11)$ & $1.07(0.97-1.19)$ \\
\hline & & & T-6 months & $1.06(0.94-1.19)$ \\
\hline & & & & T-9 weeks \\
\hline \multirow[t]{4}{*}{ B } & Observation & $0.77(0.69-0.87)$ & $0.81(0.70-0.93)$ & $0.81(0.69-0.95)$ \\
\hline & & T-12 months & $1.05(0.96-1.14)$ & $1.05(0.95-1.17)$ \\
\hline & & & T-6 months & $1.01(0.88-1.15)$ \\
\hline & & & & T-9 weeks \\
\hline \multirow[t]{4}{*}{ C } & Observation & $0.87(0.83-0.91)$ & $0.90(0.83-0.98)$ & $0.83(0.71-0.98)$ \\
\hline & & T-12 months & $1.03(0.97-1.11)$ & $0.96(0.81-1.12)$ \\
\hline & & & T-6 months & $0.92(0.77-1.10)$ \\
\hline & & & & T-9 weeks \\
\hline
\end{tabular}

Figure S7 Subgroup and sensitivity analyses in early breast cancer based on the lymph node status in the network meta-analysis. The columns were compared with the rows. Numbers in parentheses represent the $95 \%$ CI. HRs with P values $<0.05$ were considered statistically significant (red). Subgroup analysis was conducted in node-positive early breast cancer (A) and node-negative early breast cancer (B). (C) A sensitivity analysis was performed in lymph node-positive early breast cancer based on the number of positive lymph nodes $(\geq 1,1-3$ and $\geq 4$ lymph positive nodes). $\mathrm{T}$, trastuzumab.

\begin{tabular}{|c|c|c|c|c|c|}
\hline \multirow[t]{5}{*}{ A } & Observation & $0.85(0.81-0.89)$ & $0.90(0.84-0.95)$ & $0.87(0.80-0.95)$ & $0.93(0.84-1.04)$ \\
\hline & & T-12 months & $0.95(0.90-1.01)$ & $1.02(0.96-1.10)$ & $1.10(1.00-1.21)$ \\
\hline & & & T-24 months & $0.97(0.88-1.07)$ & $1.04(0.93-1.18)$ \\
\hline & & & & T-6 months & $1.08(0.95-1.21)$ \\
\hline & & & & & T-9 weeks \\
\hline \multirow[t]{5}{*}{ B } & Observation & $0.82(0.78-0.86)$ & $0.83(0.78-0.88)$ & $0.88(0.80-0.95)$ & $0.95(0.84-1.08)$ \\
\hline & & T-12 months & $1.01(0.95-1.07)$ & $1.07(1.00-1.15)$ & $1.17(1.04-1.31)$ \\
\hline & & & T-24 months & $1.06(0.96-1.16)$ & $1.15(1.01-1.32)$ \\
\hline & & & & T-6 months & $1.09(0.95-1.25)$ \\
\hline & & & & & T-9 weeks \\
\hline \multirow[t]{5}{*}{$\mathrm{C}$} & Observation & $0.85(0.81-0.89)$ & $0.90(0.84-0.96)$ & $0.86(0.79-0.94)$ & $0.90(0.75-1.08)$ \\
\hline & & $\mathrm{T}-12$ months & $1.05(0.98-1.13)$ & $1.01(0.95-1.09)$ & $1.06(0.89-1.26)$ \\
\hline & & & T-24 months & $0.96(0.87-1.06)$ & $1.01(0.84-1.22)$ \\
\hline & & & & T-6 months & $1.05(0.87-1.27)$ \\
\hline & & & & & T-9 weeks \\
\hline \multirow[t]{5}{*}{$\mathrm{D}$} & Observation & $0.82(0.78-0.86)$ & $0.83(0.78-0.88)$ & $0.87(0.80-0.95)$ & $0.85(0.68-1.06)$ \\
\hline & & T-12 months & $1.01(0.95-1.07)$ & $1.07(0.99-1.15)$ & $1.05(0.84-1.23)$ \\
\hline & & & T-24 months & $1.06(0.96-1.16)$ & $1.03(0.82-1.29)$ \\
\hline & & & & T-6 months & $0.98(0.78-1.23)$ \\
\hline & & & & & T-9 weeks \\
\hline
\end{tabular}

Figure S8 Subgroup and sensitivity analyses for disease-free survival in early breast cancer based on the hormone receptor status in the network meta-analysis. The columns were compared with the rows. Numbers in parentheses represent the $95 \%$ CI. HRs with P values $<0.05$ were considered statistically significant (red). Subgroup analysis for disease-free survival was conducted in hormone receptor-positive early breast cancer (A) and hormone receptor-negative early breast cancer patients (B). A sensitivity analysis was performed in hormone receptor-positive (C) and hormone receptor-negative (D) early breast cancer after excluding the trials that reported estrogen receptor and progesterone receptor status. $T$, trastuzumab. 
Table S1 Summary of the confidence in each comparison and ranking

\begin{tabular}{|c|c|c|c|}
\hline Comparison & Nature of the evidence & Confidence & Downgrading due to \\
\hline T-24 months vs. observation & Mixed & Moderate & Imprecision \\
\hline T-9 weeks vs. observation & Mixed & Moderate & Imprecision \\
\hline T-24 months vs. T-12 months & Mixed & Moderate & Imprecision \\
\hline T-9 weeks vs. T-12 months & Mixed & Moderate & Inconsistency \\
\hline T-12 weeks vs. T-12 months & Mixed & Moderate & Imprecision \\
\hline T-6 months vs. observation & Indirect & Moderate & Imprecision \\
\hline T-12 weeks vs. observation & Indirect & Moderate & Imprecision \\
\hline T-24 months vs. T-9 weeks & Indirect & Moderate & Imprecision \\
\hline T-6 months vs. T- 12 weeks & Indirect & Moderate & Imprecision \\
\hline T-6 months vs. T- 9 weeks & Indirect & Moderate & Imprecision \\
\hline T-12 weeks vs. T-9 weeks & Indirect & Moderate & Imprecision \\
\hline Ranking of treatments & - & Moderate & Imprecision \\
\hline
\end{tabular}

$\mathrm{T}$, trastuzumab. 